\title{
Automatic Design of Soft Dielectric Elastomer Actuators With Optimal Spatial Electric Fields
}

Chen, Feifei; Liu, Kun; Wang, Yiqiang; Zou, Jiang; Gu, Guoying; Zhu, Xiangyang

Published in:

IEEE Transactions on Robotics

Link to article, DOI:

10.1109/TRO.2019.2920108

Publication date:

2019

Document Version

Peer reviewed version

Link back to DTU Orbit

Citation (APA):

Chen, F., Liu, K., Wang, Y., Zou, J., Gu, G., \& Zhu, X. (2019). Automatic Design of Soft Dielectric Elastomer Actuators With Optimal Spatial Electric Fields. IEEE Transactions on Robotics, 35(5), 1150-1165.

https://doi.org/10.1109/TRO.2019.2920108

\section{General rights}

Copyright and moral rights for the publications made accessible in the public portal are retained by the authors and/or other copyright owners and it is a condition of accessing publications that users recognise and abide by the legal requirements associated with these rights.

- Users may download and print one copy of any publication from the public portal for the purpose of private study or research.

- You may not further distribute the material or use it for any profit-making activity or commercial gain

- You may freely distribute the URL identifying the publication in the public portal 


\title{
Automatic Design of Soft Dielectric Elastomer Actuators with Optimal Spatial Electric Fields
}

\author{
Feifei Chen, Member, IEEE, Kun Liu, Yiqiang Wang, Jiang Zou, Guoying Gu, Member, IEEE, \\ and Xiangyang Zhu, Member, IEEE
}

\begin{abstract}
Dielectric elastomer actuators (DEAs) are a promising actuation technology in soft robotics owing to their large voltage-induced deformation and rapid response. However, most existing DEA design paradigms are empirical or intuitive, lacking mathematical modeling and optimization methodology to exploit their actuation capabilities for prescribed motion tasks. In this paper, we present an automatic design methodology to maximize the concerned displacement(s) of DEAs by topology optimization of the applied spatial electric fields (SEFs). Our method is enabled by integrating the freeform SEF profile captured by implicit level sets, and the constitutive model of DEAs incorporating geometric and material nonlinearities and the electromechanical coupling effect, into a gradient-based optimizer. We implement our method for motions of single and multiple degrees of freedom (DOFs) of planar DEAs, and the optimized SEFs have been found to improve the output displacements by more than $75 \%$ compared with their intuitive counterparts. We further demonstrate a proof-of-concept application in which our designed two-DOFs DEAs can actively drive various host structures to shape-morph from flat sheets to desired three dimensional configurations. Overall, our work represents the first step toward automatic design of soft DEAs for diverse potential applications in soft machines and robots.
\end{abstract}

Index Terms-dielectric elastomer actuators, topology optimization, soft robotics, level sets.

\section{INTRODUCTION}

$\mathbf{S}$ OFT robots made of compliant materials, have gained increasing attention recently due to their inherent flexibility and adaptability to work in unstructured environment [1]-[5]. Soft actuators are the key enabling component for motion generation in such applications. During the past decade, broad categories of soft actuators have been developed, including pneumatic and fluidic actuators [6]-[10], shape memory polymers [11], [12], etc. Among them, dielectric elastomer actuators (DEAs), capable of deforming in response to electric fields, are promising owing to their interesting attributes such as large voltage-induced deformation, rapid response and high energy density [13]-[17]. Most recently, a rich repertoire of soft machines and robots based on DEAs have been reported,

This work was supported in part by the National Natural Science Foundation of China under Grant 51622506, 51620105002, and 91848204, and was sponsored by Shanghai Sailing Program (19YF1422900). (Corresponding authors: Guoying $\mathrm{Gu}$ and Xiangyang Zhu).

Feifei Chen, Kun Liu, Jiang Zou, Guoying Gu and Xiangyang Zhu are with State Key Laboratory of Mechanical System and Vibration, Shanghai Jiao Tong University, and Robotics Institute, School of Mechanical Engineering, Shanghai Jiao Tong University, Shanghai 200240, China. (email: ffchen@sjtu.edu.cn; kianliu@sjtu.edu.cn; sj_ustb@sjtu.edu.cn; guguoying@sjtu.edu.cn; mexyzhu@sjtu.edu.cn).

Yiqiang Wang is with Department of Mechanical Engineering, Technical University of Denmark, 2800 Lyngby, Denmark (email: ywang@mek.dtu.dk). such as grippers [18]-[20], bioinspired locomotive robots [21]-[25], tactile displays [26]-[28], dual-stiffness origami [29], cell stretchers [30] and medical bandages [31].

A DEA is typically made of an elastomer membrane coated with electrodes and it works as follows: when an external electric field is applied to a DEA, due to coulombic attraction effects, accumulation of opposite charges on the two sides of the membrane leads the elastomer to shrink in thickness and to expand in area [32]. The motion range of DEAs induced by the electric field is key to enabling the wide range of applications mentioned above. There are a number of factors that concurrently determine the deformed configurations of a DEA, including the prestretch, the frame used to support the DEA, and the applied electric field. In this paper, we consider planar DEAs and focus on the effect of the electric field on the deformation of DEAs.

Related to large deformation, the input spatial electric field (SEF) modulates the output displacement field in a highly nonlinear manner due to the geometric and material nonlinearities and the electromechanical coupling effect. There have been some intuitive designs of SEFs for specific applications, e.g. active shape control of inflated DEAs [33] and a DEAbased walking robot with multiple degrees of freedom (DOFs) [34]. Hajiesmaili and Clarke [35] recently realized reconfigurable shape-morphing of multi-layer dielectric elastomers, by patterning customized electric fields in each layer. In spite of these intuitive designs, the role of the freeform SEF in shaping the deformed configuration of DEAs has not been well investigated or understood.

The SEF profile in practice is embodied in the pattern of the compliant electrodes that are seamlessly attached to DEAs. Upon electric activation, the electrodes compliantly deform with the host DEA, undergoing a highly nonlinear electromechanical response. The SEF profile keeps changing as the DEA deforms, making it elusive to design the initial electrode pattern without direct knowledge of the deformation that will be induced. The intuitive design paradigm encounters this challenge since our intuitions are usually based on the undeformed state of DEAs. Furthermore, even if the induced deformation is known, one still has difficulty in developing a better SEF profile. Therefore, a comprehensive design strategy necessitates a modeling-simulation-optimization process.

In this paper, we propose a computational design methodology to effectively automate the challenging problem of designing SEFs for planar DEAs. We develop an optimization procedure to generate optimal SEFs for DEAs, aiming to accomplish prescribed motion tasks that can be mathematically stated in 
terms of directional displacements of point(s) of interest. This methodology is enabled by integrating the geometric profile of freeform SEFs and nonlinear material properties of DEAs into a gradient-based topology optimizer. First, to allow for and accurately describe arbitrary SEFs in a mathematical sense, we introduce an implicit geometric representation model by level sets, which can also naturally accommodate the topological change of the SEF during optimization. Second, the electromechanical response of DEAs induced by the current freeform SEF can be numerically analyzed based on the constitutive model of DEAs. Most important, the SEF profile subject to optimization is iteratively updated toward the steepest descent direction by conducting an adjoint sensitivity analysis, until an optimal design is evolved. Herein, the sensitivity refers to the shape derivative of the target motion with respect to the SEF profile, which incorporates material properties of DEAs.

We validate our computational method by creating SEFs targeting at DEAs' motions of single and multiple DOFs. The numerical and experimental results consistently show that our designed optimal DEAs well outperform their intuitive counterparts, and the superiority may grow pronounced to be more than $75 \%$ as the applied voltage increases. More importantly, our design method can readily handle complex tasks without additional difficulties, e.g. to maximize displacements of multiple points of interest by incorporating multiple objectives into the optimization model, while their intuitive counterpart designs can be hard to conceive. Finally, we demonstrate a proof-of-concept application in which the automatically optimized DEA is used to trigger shape-morphing of various compliant host structures from planar sheets to desired three dimensional configurations.

In a general sense, our approach paves the way for systematic design of DEAs based on mathematics and optimization theories, and gains remarkable advantages as follows for creating high-performance DEAs by leveraging the geometric flexibility of the SEFs.

1) The entire design process demands no prior knowledge of the spatial arrangement of the electric fields, and therefore it guarantees a sufficiently large design space.

2) We use a large number of design variables to search for the optimal designs within the design space, and our gradient-driven optimization algorithms make it efficient to converge to feasible solutions. Indeed, the obtained novel designs remarkably outperform their intuitive counterparts.

3) Our design approach can be readily generalized to design DEAs with increased complexities in terms of the actuator geometry or motion specifications that in contrast may quickly render intuitive designs ineffective.

4) Our design approach does not necessitate any additional complex mechanical design or fabrication process. We embed the design complexity in the amorphous SEF profile that can be easily implemented in practice by arranging the optimized electrodes through masks.

The remainder of this paper is organized as follows. Section II introduces the implicit geometric model based on level sets for capturing amorphous SEFs. Section III derives the state

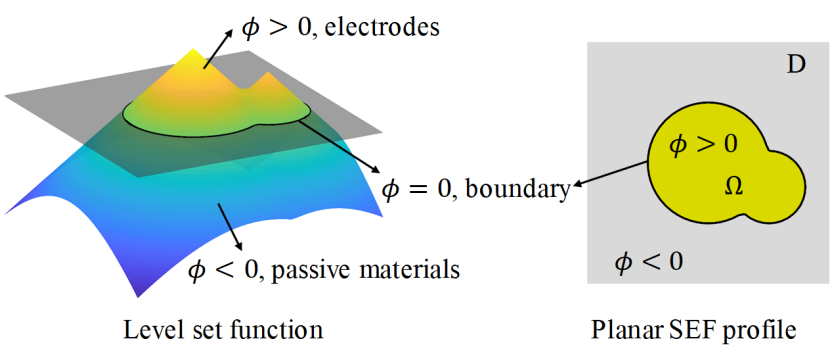

Fig. 1: Representation of a planar SEF profile with a single level set function in the form of a signed distance function.

equation of a DEA activated with arbitrary SEFs based on the constitutive model. Section IV introduces the topology optimization of SEF profiles, targeting at prescribed motion tasks of DEAs. Section V numerically implements the design approach for motions of single and multiple DOFs of planar DEAs, and the optimized designs are experimentally verified in comparison with their intuitive counterparts in Section VI. Section VII shows proof-of-concept applications of the optimized DEAs. Discussions and conclusions are finally drawn in Section VIII.

\section{IMPLICIT GeOMETRIC MODEL}

Level sets have been widely used as a versatile tool for evolving amorphous shapes [36], and have been well embedded in classical topology optimization approaches [37], [38]. The level-set model makes it convenient to follow shape and topological changes, e.g., when a shape splits, develops holes, or the reverse of these operations.

In this paper, we adopt a level set model to implicitly capture the freeform SEF profile applied to DEAs. We consider DEAs in their most basic form, i.e. an elastomeric dielectric sheet sandwiched between a pair of compliant electrodes. We construct a three dimensional level set function $\phi$ with its zero contours representing the SEF profile, as shown in Fig. 1, stated by

$$
\begin{cases}\phi(\mathbf{X}, t)>0, & \forall \mathbf{X} \in \Omega \\ \phi(\mathbf{X}, t)=0, & \forall \mathbf{X} \in \Gamma \\ \phi(\mathbf{X}, t)<0, & \forall \mathbf{X} \in \mathrm{D} /(\Omega \cup \Gamma)\end{cases}
$$

where $\mathrm{D}$ denotes the design domain spanned by the DEA itself, $\Omega$ denotes the area applied with SEF, i.e. the electrode pattern, $\Gamma$ denotes the SEF boundaries, $\mathbf{X} \in \mathrm{D}$ represents the coordinates of a point within the design domain, and $t$ denotes a pseudo-time for evolution of the level set function.

Remark 1. The level set function is typically defined as a signed distance function, i.e. the function value at a given point is the minimal distance from the boundary in question, with the sign determined by whether the point is in $\Omega$. A signed distance function is differentiable almost everywhere and its gradient satisfies the eikonal equation.

Evolution of the SEF profile is realized by updating the zero contour of the level set function implicitly. By differentiating both sides of $\phi(\mathbf{X}, t)=0$ with respect to the time and 


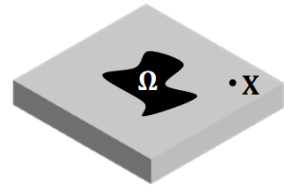

(a)

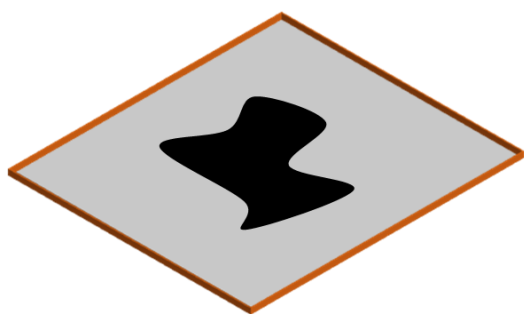

(b)

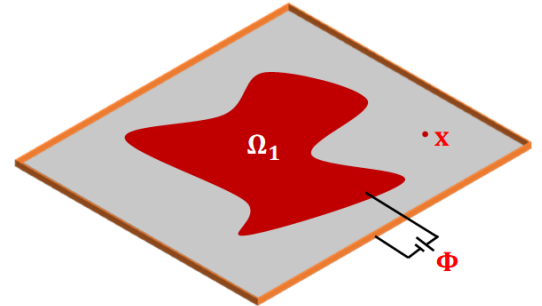

(c)

Fig. 2: Schematic of a DEA: (a) the reference state with an electrode pattern $\Omega$, (b) the prestretched state, and (c) a current state subject to a voltage $\Phi$, resulting in the deformed electrode pattern $\Omega_{1}$.

applying the chain rule, we obtain the so-called HamiltonJacobi equation,

$$
\frac{\partial \phi(\mathbf{X}, t)}{\partial t}+\nabla \phi(\mathbf{X}, t) \cdot \mathbf{V}=0
$$

where $\mathbf{V}=\mathrm{d} \mathbf{X} / \mathrm{d} t$ is the moving velocity of point $\mathbf{X}$. It is known that the shape change is only related to the normal movement of the boundaries. Hence, (2) can be restated by,

$$
\frac{\partial \phi(\mathbf{X}, t)}{\partial t}=-|\nabla \phi(\mathbf{X}, t)| V_{n}
$$

where $V_{n}=\mathbf{V} \cdot \mathbf{n}$ refers to the normal velocity at point $\mathbf{X}$, with $\mathbf{n}=-\nabla \phi /|\nabla \phi|$ the normal direction in which $\phi$ decreases most. The normal velocity will provide the gradient information to update the SEF profile in the optimization process. Eq. (3) supervises the evolvement of the level set function for given moving velocity on the frontier boundaries, in order to achieve shape and topology changes, and it mathematically defines an initial value problem for the time-dependent level set function.

Remark 2. The partial differential equation (3) is solved by using essentially non-oscillatory finite difference strategy. To ensure numerical stability, the movement distance within time $t$ should satisfy the Courant-Friedrichs-Lewy condition. The reader may refer to [39] for more implementation details.

\section{EleCtromechanicAl ANALYSis of DEAs}

In this section, we will introduce the constitutive model of DEAs that incorporates the hyperelasticity and the electromechanical coupling effect, and formulate the state equation of DEAs activated with SEFs of amorphous profiles.

\section{A. Actuation Principle}

DEAs are typically silicon- or acrylic-based polymers, e.g., polydimethylsiloxane or the widely used Very High Bond (VHB) acrylic adhesive by 3M company [40]. Fig. 2 illustrates the principle of operation of a DEA. In the reference state, a membrane of DEA at rest is patterned with compliant electrodes (highlighted in black, denoted by $\Omega$ ). In the prestretched state, the DEA is bi-axially stretched and then is mounted to a rigid frame to maintain the prestrain. In a current state, a high voltage is applied to activate the areas patterned with electrodes, and the deformed electrode pattern is highlighted in red (denoted by $\Omega_{1}$ ).

\section{B. Constitutive Modeling}

As shown in Fig. 2, we use $\mathbf{X}$ and $\mathbf{x}$ to denote the coordinates of a particle in the dielectric membrane at the reference and current states, respectively. The induced displacement $\mathbf{u}$ and the deformation gradient $\mathbf{F}$ of the particle are defined by

$$
\begin{gathered}
\mathbf{u}=\mathbf{x}-\mathbf{X}, \\
\mathbf{F} \equiv \nabla_{0} \mathbf{x}=\mathbf{I}+\nabla_{0} \mathbf{u}
\end{gathered}
$$

where $\nabla_{0}=\frac{\partial}{\partial \mathbf{X}}$ denotes the gradient operator with respect to the reference domain, and $\mathbf{I}$ is the second-order identity tensor.

The free energy density $W$ is defined to characterize the energy density distribution of the elastic dielectric induced by the mechanical and electric work [32], stated by

$$
W=W^{u}+W^{e}
$$

with $W^{u}$ and $W^{e}$ denoting the energy densities attributed to the mechanical stretching and electrical polarization, respectively. The strain energy density of an isotropic hyperelastic material is generally defined by a function of the deformation gradient or the three invariants of the strain tensor. In this work, without loss of generality, we employ the generalized neo-Hookean model [41] to characterize the hyperelasticity and compressibility of DEAs. Herein, the mechanical free energy density $W^{u}$ is expressed in terms of the first and third regulated strain invariants of the right Cauchy-Green deformation tensor $\mathbf{B}=\mathbf{F}^{\mathrm{T}} \cdot \mathbf{F}$ (the superscript $\mathrm{T}$ denotes the transpose),

$$
W^{u}=\frac{\mu}{2}\left(\bar{I}_{1}-3\right)+\frac{\kappa}{2}\left(\bar{I}_{3}-1\right)^{2}
$$

where $\mu$ and $\kappa$ denote the initial shear modulus and bulk modulus at small stretches, respectively. The two regulated invariants are defined as follows,

$$
\bar{I}_{1}=\frac{\operatorname{trace}(\mathbf{B})}{\bar{J}^{2 / 3}}, \quad \bar{I}_{3}=\sqrt{\operatorname{det}(\mathbf{B})}=\bar{J}
$$

where $\bar{J}=\operatorname{det}(\mathbf{F})$ denotes the determinant of the deformation gradient.

Remark 3. Many other hyperelastic material models such as the Mooney-Rivlin model and the Gent model can be readily embedded into the present design framework without additional fundamental difficulties. 


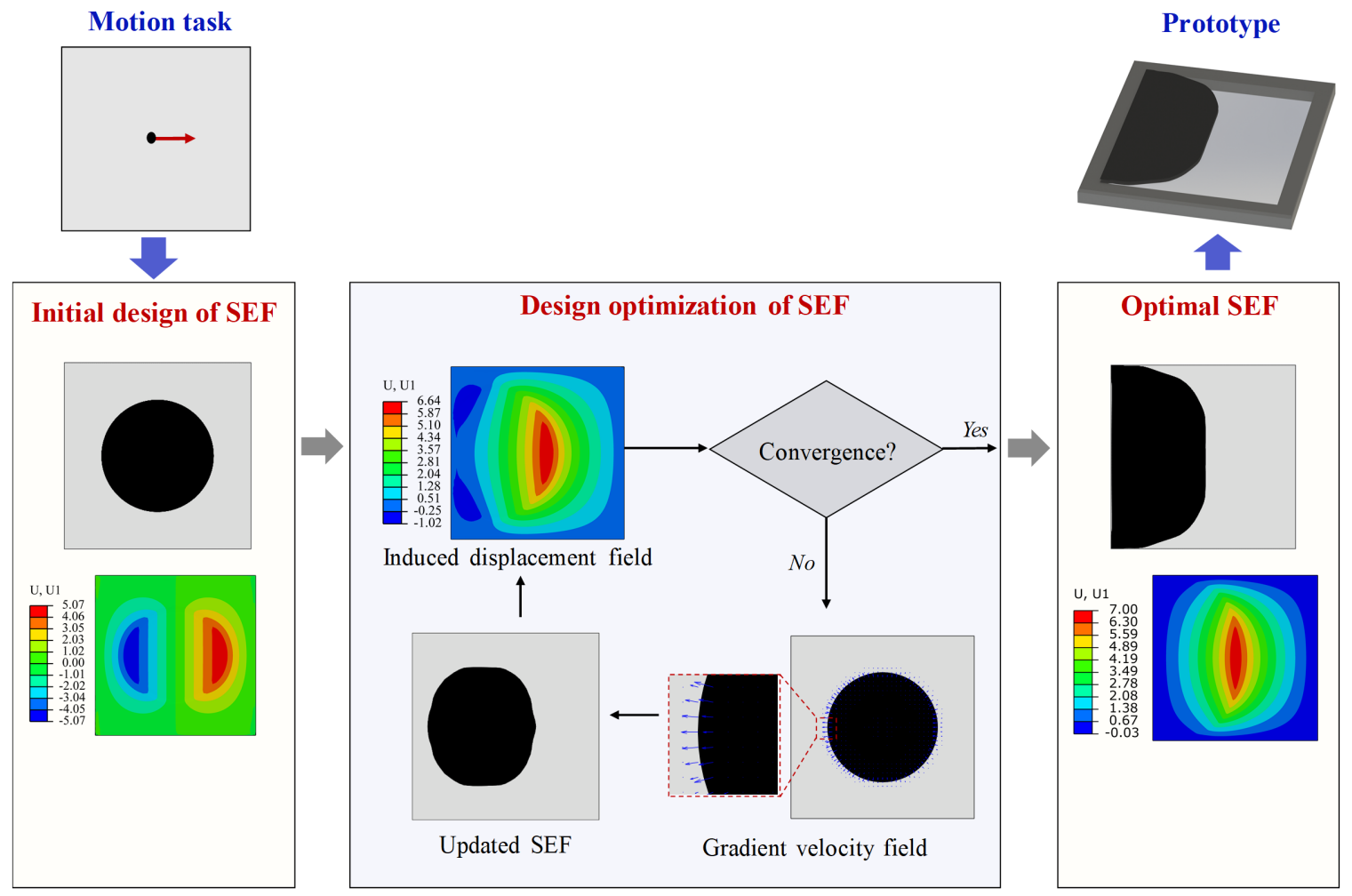

Fig. 3: Illustrative SEF design flowchart of a DEA for a specified motion task.

As developed by Suo [32], in order to make the electric field as an independent variable, we employ the so-called electrical Gibbs free energy density, with $W^{e}$ taking the following form

$$
W^{e}=-\frac{1}{2} \varepsilon \bar{J} B_{i j}^{-1} \tilde{E}_{i} \tilde{E}_{j}
$$

where $\varepsilon$ is the permittivity, the subscripts $i, j$ have values 1 , 2 , or 3 that represent the three Cartesian coordinates, and $\tilde{\mathbf{E}}$ denotes the nominal electric field defined as the gradient of the electric potential $\Phi$ by

$$
\tilde{\mathbf{E}}=\left\{\begin{array}{ll}
-\nabla_{0} \Phi, & \forall \mathbf{X} \in \Omega \cup \Gamma \\
\mathbf{0} & , \quad \forall \mathbf{X} \in \mathrm{D} /(\Omega \cup \Gamma)
\end{array} .\right.
$$

The nominal stress tensor can be derived by differentiating the electrical Gibbs free energy density with respect to the deformation gradient,

$$
\mathbf{s}=\frac{\partial W}{\partial \mathbf{F}}=\frac{\partial W^{u}}{\partial \mathbf{F}}+\frac{\partial W^{e}}{\partial \mathbf{F}}
$$

with its mechanical and electric components as follows

$$
\mathbf{s}^{u}=\frac{\partial W^{u}}{\partial \mathbf{F}}, \mathbf{s}^{e}=\frac{\partial W^{e}}{\partial \mathbf{F}}
$$

where $\mathbf{s}^{e}$ refers to the well-known Maxwell stress that physically explains the electromechanical coupling effect of DEAs. Refer to Appendix A for the specific expressions of $\mathbf{s}, \mathbf{s}^{u}$ and $\mathbf{s}^{e}$ in the tensor form.

\section{State Equation}

The state equation of the activated DEA at the equilibrium state can be established based on the reference domain, by using the principle of virtual work,

$$
\begin{aligned}
& \int_{\mathrm{D}} \mathbf{s}^{u}(\mathbf{u}): \nabla_{0} \mathbf{v} \mathrm{d} \Omega+\int_{\Omega} \mathbf{s}^{e}(\mathbf{u}, \tilde{\mathbf{E}}): \nabla_{0} \mathbf{v} \mathrm{d} \Omega=0, \forall \mathbf{v} \in \bar{U} \\
& \text { subject to } \quad \mathbf{u}=\overline{\mathbf{u}}, \forall \mathbf{X} \in \Gamma_{\mathrm{D}}
\end{aligned}
$$

where $\mathbf{v}$ is the virtual displacement field that belongs to the kinematically admissible displacement space $\bar{U}$. To capture the process of prestretch, a non-zero displacement constraint $\overline{\mathbf{u}}$ is imposed along the external boundaries of the design domain, denoted by $\Gamma_{\mathrm{D}}$. For convenience of expression, we define the left hand side of the state equation (13) to be $a(\mathbf{u}, \mathbf{v}, \Omega)$.

\section{Optimal Design of SeF Profile}

\section{A. Design Workflow}

To fully equip a DEA with actuation capabilities for a prescribed motion task, maximization of the induced displacements at concerned regions is pursued as the design objective. The design variable is the freeform SEF profile applied to the planar DEA, implicitly captured by level sets, and undergoes a topology optimization procedure.

A general end-to-end design flowchart is illustrated in Fig. 3. For a prescribed motion task, the design process starts from an initial SEF that can be randomly generated; then nonlinear finite element analysis is conducted to solve (13) to yield the displacement field in the current configuration, from which the 
design objective in terms of the displacement(s) in question can be evaluated; third, a gradient velocity field is calculated to guide the moving directions of the SEF boundaries, through carrying out an adjoint sensitivity analysis; consequently, the SEF profile is updated by solving (3). The above steps are repeated until the prescribed convergence criteria are met.

\section{B. Optimization Model}

The optimization model is formulated by,

$$
\begin{array}{ll}
\min _{\Omega} & J=-\sum_{i=1}^{M} \mathbf{u}\left(\mathbf{p}_{i}\right) \cdot \mathbf{T}_{i} \\
\text { subject to } & a(\mathbf{u}, \mathbf{v}, \Omega)=0, \forall \mathbf{v} \in \bar{U}, \\
& \mathbf{u}=\overline{\mathbf{u}}, \forall \mathbf{X} \in \Gamma_{\mathrm{D}}, \\
& \varsigma-f|\mathrm{D}|=0
\end{array}
$$

where $J$ is the design objective, $M \geq 1$ is the total number of concerned points $\mathbf{p}_{i} ; \mathbf{T}_{i}$ is the transformation matrix to project the displacement $\mathbf{u}\left(\mathbf{p}_{i}\right)$ in the concerned direction; $\varsigma=\int_{\Omega} \mathrm{d} \Omega$ defines the electrode area, and the area constraint $\varsigma-f|D|=0$ plays regularization roles when performing optimization, with $f$ and $|\mathrm{D}|$ the given area fraction for the SEF and the total area of the planar design domain.

Remark 4. The present optimization problem is highly loaddependent in the sense that the electric loading by applying the SEF will change with the deformation of DEAs, partly due to the geometric change of the SEF profile and partly due to the increasing magnitude of the electric field.

\section{Sensitivity Analysis}

We carry out a shape sensitivity analysis to estimate how the objective and constraint functions change with an SEF variation, which is also called shape derivative. A radical challenge is that the shape derivative of the displacement field is involved but elusive to evaluate. Hence, the adjoint sensitivity analysis method is adopted to eliminate the shape derivative of the displacement field. The first step is to formulate the Lagrangian of the optimization problem,

$$
L=J+a(\mathbf{u}, \mathbf{w}, \Omega)+\lambda(\varsigma-f|\mathrm{D}|)
$$

where a virtual displacement $\mathbf{w} \in \bar{U}$ is introduced and will then be identified as the adjoint displacement, and $\lambda$ denotes the Lagrange multiplier. After lengthy but straightforward derivations as narrated in Appendix B, the shape derivative of the Lagrangian reads

$$
\begin{aligned}
& \dot{L}=\int_{\Omega}\left[-\mathbf{C}: \nabla_{0} \mathbf{u} \nabla_{0} \mathbf{V}: \nabla_{0} \mathbf{w}-\mathbf{s}: \nabla_{0} \mathbf{w} \nabla_{0} \mathbf{V}\right. \\
& \left.+\left(\mathbf{s}: \nabla_{0} \mathbf{w}+\lambda\right) \nabla_{0} \cdot \mathbf{V}\right] \mathrm{d} \Omega
\end{aligned}
$$

where $\mathbf{C}=\partial \mathbf{s} / \partial \mathbf{F}$ is a fourth-order material constitutive tensor as expressed in Appendix A.

\section{Design Velocity Selection}

The shape derivative (16) involves gradient and divergence terms of the velocity field, making it difficult to explicitly select a proper normal velocity field for ensuring descending directions. Instead, as narrated in Appendix C, we discretize (16) on a regular mesh and then select a class of normal velocities at the grid points, denoted by $\mathbf{V}_{n}$,

$$
\begin{array}{r}
\mathbf{V}_{n}^{\mathrm{T}}=\underset{e=1}{\stackrel{N_{e}}{\mathrm{~A}} \int_{0 \Omega^{e}}}\left[\mathbf{w}_{\nabla}^{\mathrm{T}}\left(\mathbf{C}^{*}\right)^{\mathrm{T}} \tilde{\mathbf{u}} \mathbf{H}+\mathbf{s}_{V}^{\mathrm{T}} \tilde{\mathbf{u}} \mathbf{H}\right. \\
\left.-\left(\mathbf{s}_{V}^{\mathrm{T}} \mathbf{w}_{\nabla}+\lambda\right) \Lambda^{\mathrm{T}}\right] \mathbf{n}^{e} \mathrm{~d} \Omega
\end{array}
$$

where the related terms are defined in Appendix C.

\section{E. Optimization Implementation}

In the numerical implementation, a regular design domain is discretized using bilinear quadrilateral-4 elements and the level set function is assigned at the grid nodes. We use the common bilinear shape function to interpolate the nodal level sets, in order to construct a continuous level set field in the design domain. Within the total Lagrange framework, nonlinear finite element analysis is conducted in MATLAB to obtain the nodal displacements, undergoing an iterative process using the Newton-Raphson method. The adjoint displacement field is directly obtained by solving the linear equation (32). After obtaining the displacement and the adjoint displacement, the design velocity can be calculated using (17) in a straightforward manner, and then the level set function is updated by solving (3).

During the optimization process, the Lagrange multiplier is iteratively updated by the following strategy,

$$
\lambda_{k+1}=\lambda_{k}+\xi_{k}\left(\varsigma_{k}-f|\mathrm{D}|\right), \xi_{k+1}=\gamma \xi_{k}, k=1,2, . ., N_{i t e r}
$$

where $\xi_{k}$ denotes the penalization factor at the $k$ th step, $\gamma \geq 1$ is an expansion parameter and $N_{i t e r}=1000$ is the prescribed maximum iteration step of the nonlinear optimization. The optimization algorithm terminates until the relative difference of the objective function values between two successive iterations is lower than $10^{-3}$ within at least 150 iteration steps.

\section{Simulation}

In this section, we will numerically investigate three cases including motion tasks of single and multiple DOFs. We demonstrate the effectiveness of our design strategy to produce the optimal DEAs in terms of the SEF profile and compare the performances with their intuitive counterparts.

\section{A. Parameter Setting}

We use VHB 4910 for prototyping the DEAs. A $40 \mathrm{~mm} \times$ $40 \mathrm{~mm} \times 1 \mathrm{~mm}$ membrane at rest is taken as the design domain. The material parameters in the hyperelastic Neo-Hookean model are fitted to be $\mu=45 \mathrm{kPa}$ and $\kappa=2.35 \mathrm{MPa}$. The prestretch is set to be $3 \times 3$ times based on our experimental experience. The permittivity is set to be $\varepsilon=4.0 \times 10^{-11} \mathrm{~F} / \mathrm{m}$ [28]. The applied voltage $\Phi$ is prescribed to be $6 \mathrm{kV}$. 


\section{B. Case I: Motion of a Single Point}

1) Optimization Problem: For proof of concept, we first investigate a simple motion task, with reference to Fig. 4(a), where point $O$ in the Cartesian coordinate $X O Y$ is expected to move in the horizontal direction. The design objective is stated by

$$
\min _{\Omega} J=-\mathbf{u}_{O} \cdot \mathbf{T}_{O}
$$

where the $\mathbf{u}_{O}$ denotes the nodal displacement of point $O$, and the transformation vector is $\mathbf{T}_{O}=[1,0]^{\mathrm{T}}$.

2) Optimization Results: One may easily figure out one intuitive design where the SEF is distributed on the left half part of the DEA, as shown in Fig. 4(b). However, our optimization result [see Fig. 4(c)] creates an SEF with freeform boundaries of different shape from the intuitive design. The iteration history of the optimization process is shown in Fig. 4(d). Specifically, the optimization process starts with an initial design of the SEF that contains many small circles to allow for sufficient geometric flexibility for potential topological evolutions. Fig. 4(d) also shows the intermediate and final SEFs in terms of the planar profiles and their corresponding level set functions. Topological changes that the small circles merge into an integral shape are observed. Refer to the supplemental video $\mathrm{S} 1$ for the dynamic evolution process.

Remark 5. The computational efficiency of the optimization process relies on the mesh resolution for solving the nonlinear state equation. Roughly speaking, the finite element analysis takes over $90 \%$ computing time. To accelerate the computation, we used the displacement field in the previous step as the initial guess to obtain the current displacement field through Newton-Raphson iterations. This strategy can save the analysis time by more than $80 \%$. In our testing cases with a regular mesh including $40 \times 40$ elements, one optimization step takes around 30 seconds.

To evaluate the actuation performance, we implement the final optimized design and the corresponding intuitive design in the commercial finite element analysis software ABAQUS, where a previous user-defined material subroutine for DEAs is adopted with the material properties modified according to our experimental characterizations [42]. Here, the 8-node quadratic hexahedral element is used to conduct the static simulation. The simulation results show that the optimized SEF enlarges the horizontal displacement of point $O$ by an extent of more than 14\%, as shown by Figs. 4(e) and (f). This finding demonstrates the necessity of performing design optimization of the SEFs since our intuition or experience is limited even in such a simple case.

Remark 6. Since the prestretch process is fixed, the displacement mentioned in the simulation results (as well as experimental results in the following section) refers to the component induced by the applied voltage, i.e. the displacement from the prestretched state to the activated state, while the component caused by the prestretch is automatically omitted.

3) Determination of the Area Fraction: We investigate how the optimization result depends on the area fraction. We

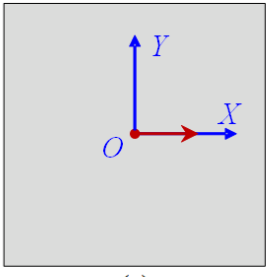

(a)

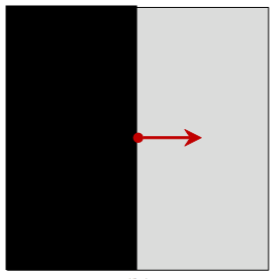

(b)

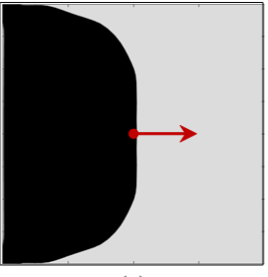

(c)

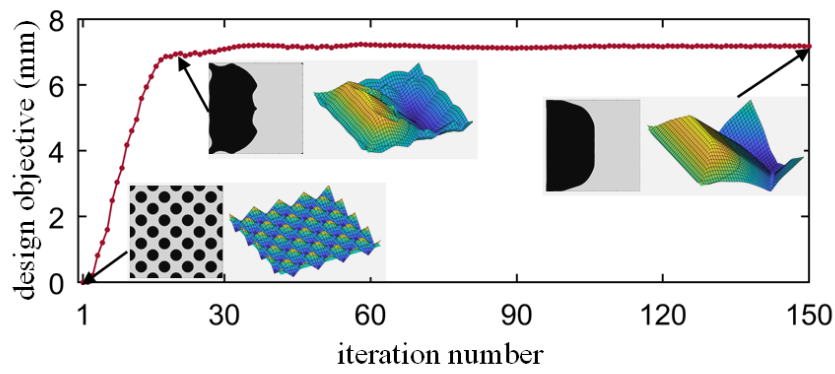

(d)

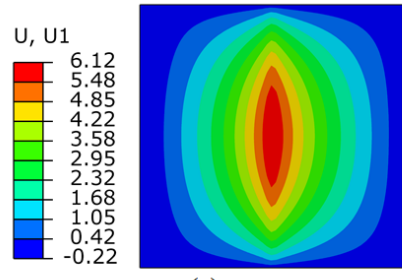

(e)

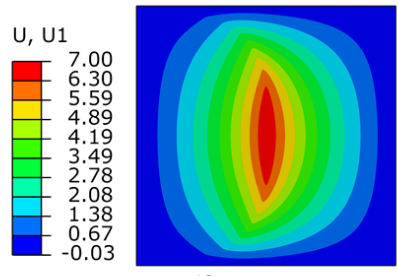

(f)
Fig. 4: Case I: (a) the design problem; (b) an intuitive design; (c) the optimized design; (d) the optimization history with insets of the initial, intermediate and optimized SEF profiles in terms of planar geometries and their corresponding level set functions; (e) the simulation result of the intuitive design with $\mathrm{U}_{1}$ the horizontal component of the displacement field; (f) the simulation result of the optimized design.

change the area fraction from 0.2 to 0.5 with an interval of 0.05 , and perform the optimization procedure for each area fraction. The optimized results and their corresponding displacements at $\Phi=6 \mathrm{kV}$ are shown in Fig. 5. When the area fraction increases from 0.2 to 0.45 , the resulting horizontal displacement of point $O$ increases gradually. That is, the DEA benefits from increased activated areas by the electric field. Herein, the growth path of the SEF profile with the increase of the area fraction implies the priority of arranging the electrodes on the dielectric membrane so as to maximally deform point $O$ horizontally. Specifically, when subjected to an electric field, the areas closer to point $O$ and less deviated from the $O X$ direction contribute more to the horizontal displacement of point $O$ and thus are activated in priority. The benefit of increasing activated areas grows marginal as the area fraction increases, e.g. when $f$ changes from 0.4 to 0.45 .

When the area fraction further increases from 0.45 to 0.5 , the performance drops. On the one hand, the positive contribution of additional activated area to the target motion is marginal. On the other hand, the additional activated area will relax the tension for the already activated areas, to some extent suppressing their deformations and thus making negative contributions to the target motion. The negative contributions 


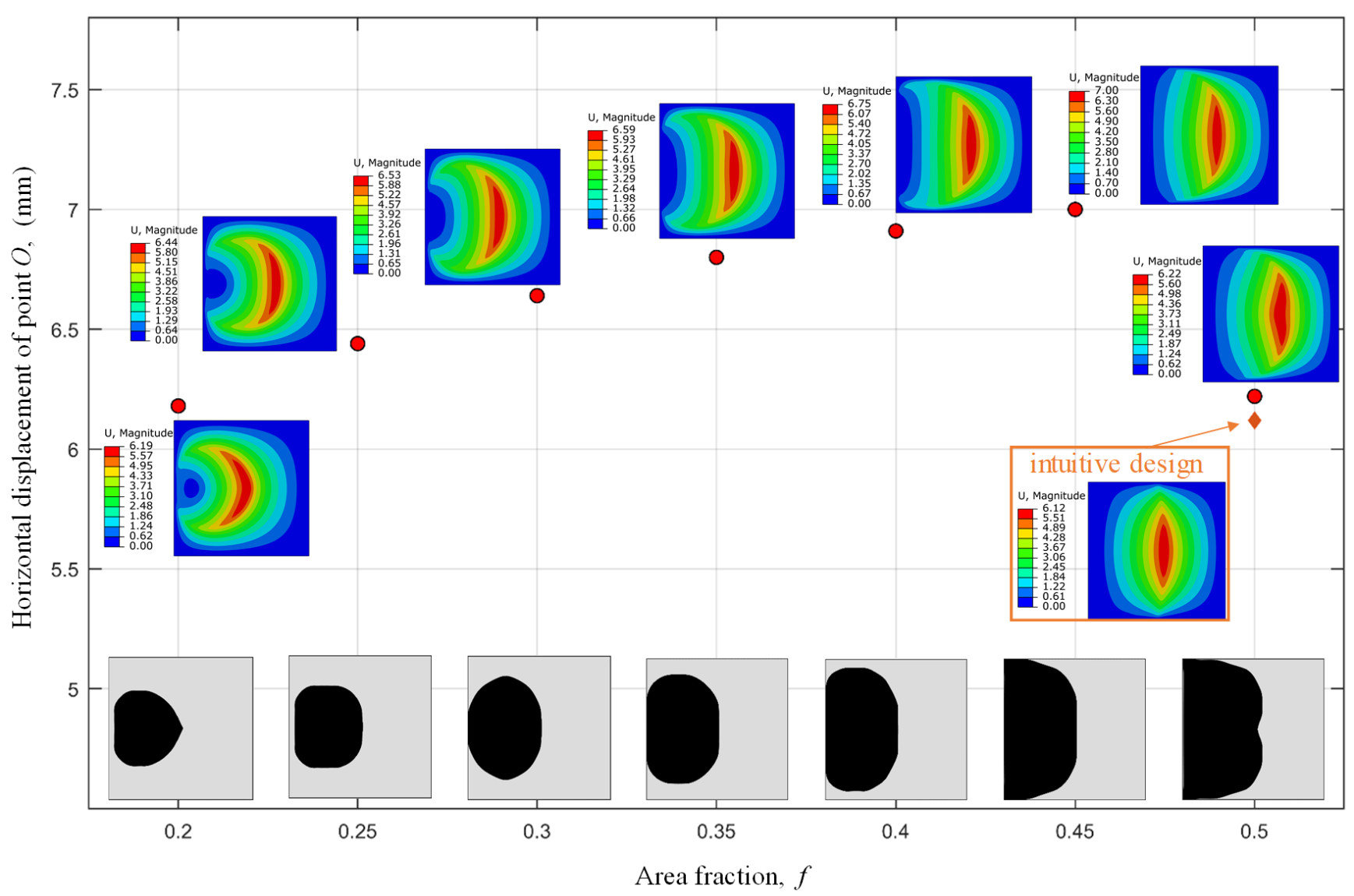

Fig. 5: The performances of the optimized DEAs of Case I, plotted on the reference configuration, vary with the area fraction. The optimized designs and their displacements at $\Phi=6 \mathrm{kV}$ are plotted as insets, in comparison with the intuitive design.

counteract the marginal positive contributions, resulting in the drop of the actuation performance. Therefore, the area fraction $f=0.45$ under which the concerned displacement peaks is selected for the final optimal design.

\section{Case II: Motion of an Arbitrary Point toward an Arbitrary Direction}

1) Optimization Problem: To extend Case I to more complicated cases, we study design problems that maximize the displacement magnitude of a randomly selected point moving in an arbitrary direction. As shown in Fig. 6(a), point $A$ is expected to move toward the prescribed direction. The objective function is then stated by,

$$
\min _{\Omega} J=-\mathbf{u}_{A} \cdot \mathbf{T}_{A}
$$

where $\mathbf{u}_{A}$ denotes the nodal displacement of point $A$, and the transformation vector is $\mathbf{T}_{A}=\left[\cos \frac{\pi}{6}, \sin \frac{\pi}{6}\right]^{\mathrm{T}}$.

2) Optimization Results: We may figure out one intuitive design of the SEF as shown in Fig. 6(b). The optimized SEF [see Fig. 6(c)] presents a different shape from the intuitive design. The optimization history is shown in Fig. 6(d) and the supplemental video $\mathrm{S} 2$. The area fraction is set to be 0.65 after numerical tests. We observe that, in the optimized design, the tangential of the deformed electrode boundary at point $A$ is perpendicular to the direction of the target motion.
Two remarkable differences are observed by comparing the optimized SEF geometry with the intuitive design. Firstly, as in Case I, some areas deviated from the prescribed direction of displacement are active in the intuitive design but are deactivated in the optimized design since their marginal positive contributions will be counteracted. Second, along the direction of the target motion, the optimized SEF profile peaks at point $A$, and as expected, the maximal displacement occurs exactly at point $A$. On the contrary, it seems that the intuitive design will always be such when any point on the same electrode boundary is investigated [see Fig. 6(b)]. In other words, the intuitive design is not specifically designed for point $A$, and thus the maximal displacement would occur on the boundary, not necessarily at point $A$.

Similar to Case I, the optimized design and the intuitive design are implemented in ABAQUS, and by coordinate rotation, we project the displacement field along the prescribed direction, as shown in Figs. 6(e) and (f). The directional displacements of point $A$ for the intuitive design and the optimized design are $4.58 \mathrm{~mm}$ and $5.80 \mathrm{~mm}$, respectively, resulting in a relative improvement of more than $25 \%$.

\section{Case III: Motion of Multiple Points for High-level Tasks}

1) Optimization Problem: It is convenient to extend the optimization problem to handle displacements of multiple 


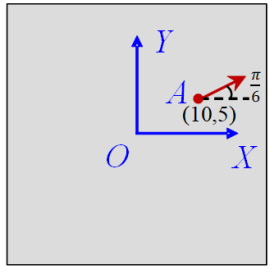

(a)

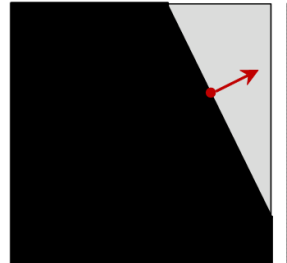

(b)

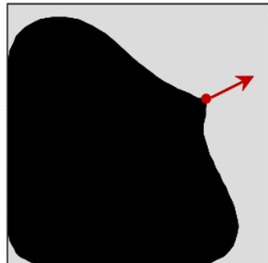

(c)

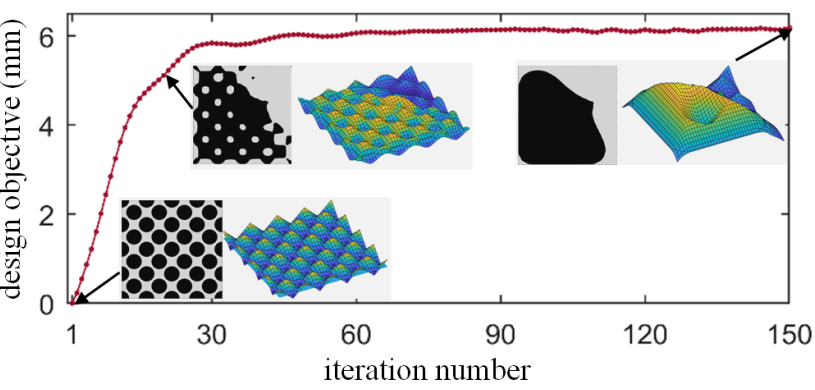

(d)

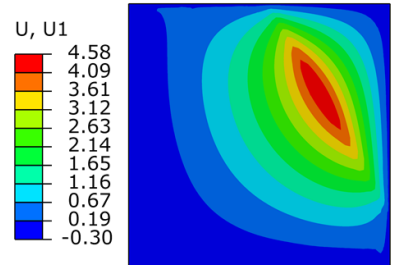

(e)

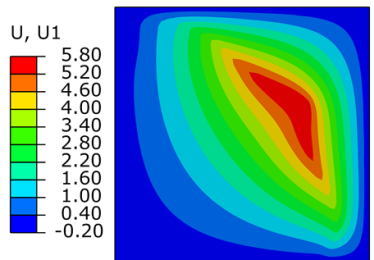

(f)
Fig. 6: Case II: (a) the design problem; (b) an intuitive design; (c) the optimized design; (d) the optimization history with insets of the initial, intermediate and optimized SEF profiles in terms of planar geometries and their corresponding level set functions; (e) the simulation result of the intuitive design with $\mathrm{U}_{1}$ the displacement field along the prescribed direction; (f) the simulation result of the optimized design.

points that are typically required in complex motion tasks of soft DEA-based robots. Motivated by a specific application for driving shape-morphing of planar sheets to desired three dimensional configurations (as will be demonstrated in Section VII), four prescribed points in the reference frame are expected to move toward the center point to induce compressive loading for triggering buckling of the planar sheets, as shown in Fig. 7(a). The design objective can be formulated to be

$$
\min _{\Omega} J=-\sum_{i=1}^{4} \mathbf{u}\left(\mathbf{p}_{i}\right) \cdot \mathbf{T}_{i}
$$

with $\mathbf{p}_{i}(i=1,2,3,4)$ the four points in question.

2) Optimization Results: As shown in Figs. 7(b) and (c), the optimized design presents quite a different configuration from its intuitive counterpart. The optimization history is shown in Fig. 7(d) and the supplemental video S3. Again, the normals of the SEF boundary at the prescribed four points each are perpendicular to the directions of the target motions. Figs. 7(e) and (f) show that the optimized design well outperforms the intuitive design in terms of the induced displacements along the prescribed directions.

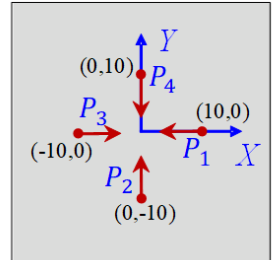

(a)

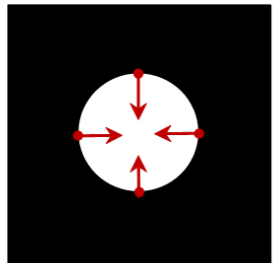

(b)

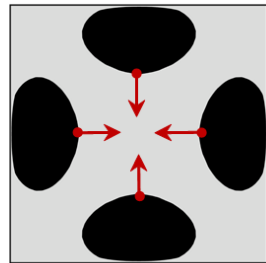

(c)

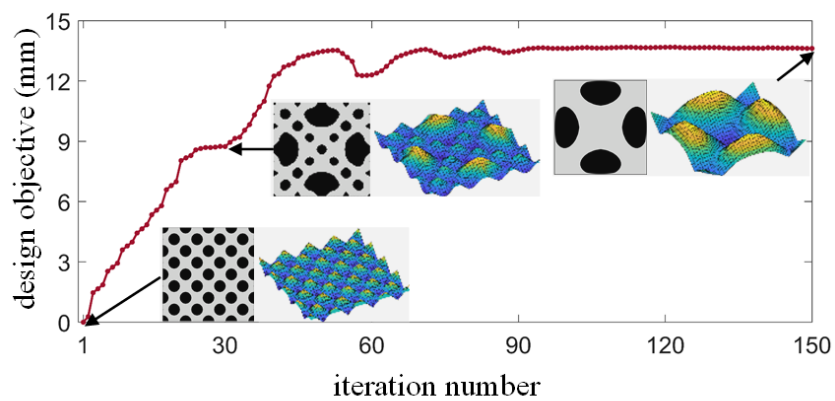

(d)

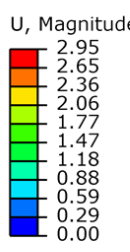

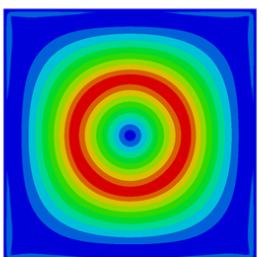

(e)

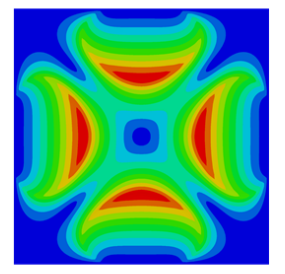

(f)
Fig. 7: Case III: (a) the design problem; (b) an intuitive design; (c) the optimized design; (d) the optimization history with insets of the initial, intermediate and optimized SEF profiles in terms of planar geometries and their corresponding level set functions; the simulation results of (e) the intuitive design and (f) the optimized design.

It is interesting to note that, we identify the area fraction of the optimal design to be 0.35 after some numerical implementations, much smaller than that of the intuitive design, i.e. 0.80. This remarkable difference again demonstrates that larger activated areas of DEAs do not necessarily lead to better performance, and instead we need to seek for the optimal arrangement of the electrodes.

We also evaluate the strain, stress and electric fields of the optimized design when subjected to a voltage of $6 \mathrm{kV}$. The results are shown in Fig. 8 in which the prestretched configuration is taken as the reference frame (denoted by $x y$ coordinates). Specifically, Figs. 8(a) and (b) plot the in-plane maximum and minimum principal nominal strains, respectively, corresponding to the square roots of the eigenvalues of the right Cauchy-Green tensor B. As expected, the maximum strain occurs within the electrode areas, reaching 0.14 . The out-of-plane strain, i.e. along the thickness direction, is captured by Fig. 8(c). It is observed that the whole electrode areas are compressed in thickness, resulting in negative strain values with magnitude up to 0.19 .

Figs. 8(d) and (e) show the in-plane maximum and minimum principal nominal stresses, respectively, corresponding to the eigenvalues of the nominal stress tensor $\mathbf{s}$. It is observed 


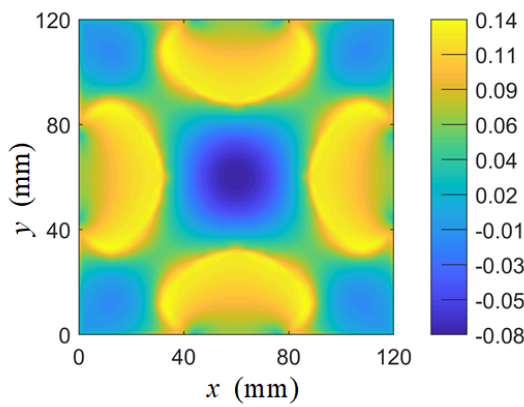

(a) In-plane NE, Max. Principal

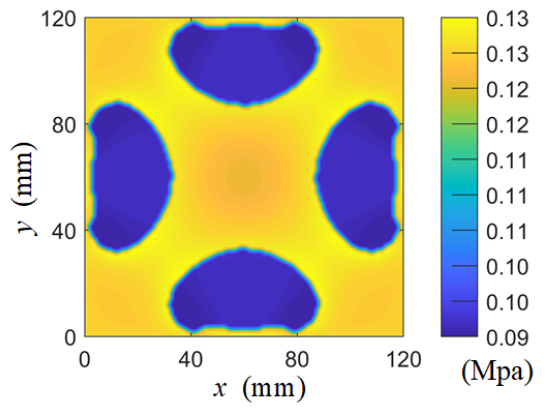

(d) In-plane NS, Max. Principal

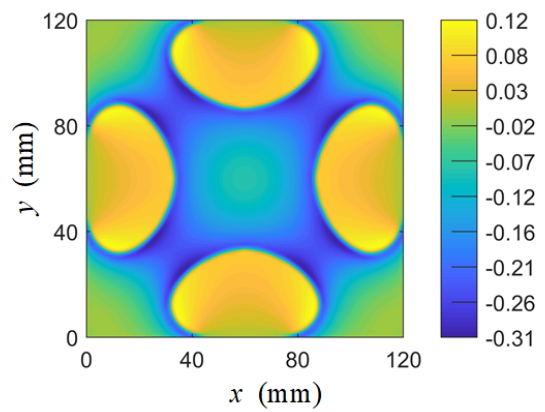

(b) In-plane NE, Min. Principal

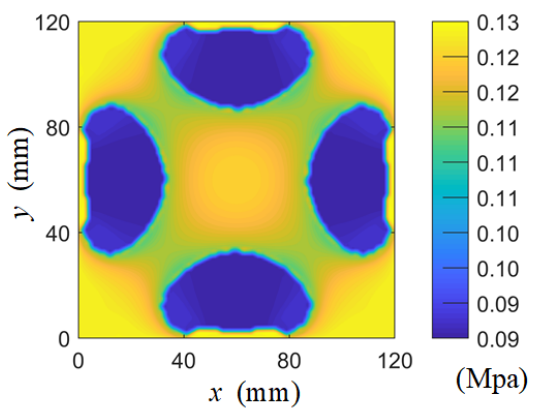

(e) In-plane NS, Min. Principal

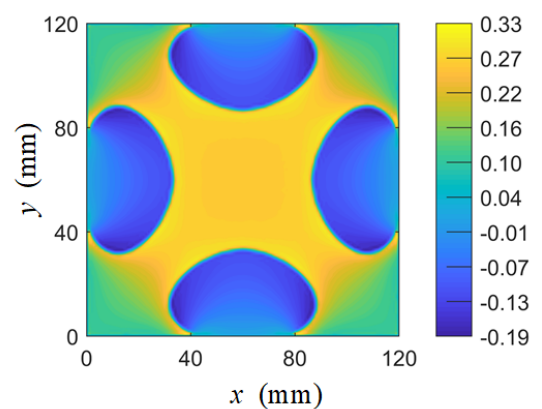

(c) Out-of-plane NE

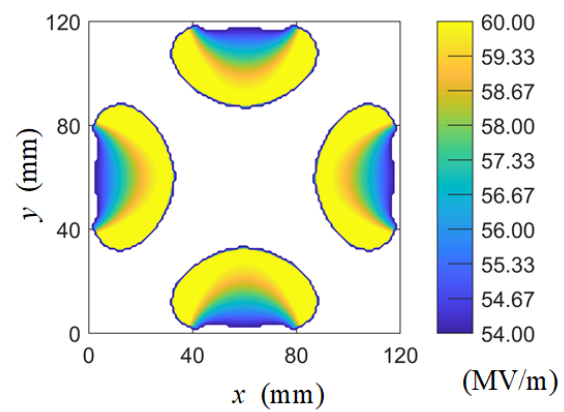

(f) Electric fields

Fig. 8: The strain, stress and electric fields of the optimized design in Case III.

that, the whole dielectric membrane is subject to tensile stress, and thus loss of tension, a typical material failure mode, does not occur. The underlying reason is that we have provided the membrane with a prestretch of $3 \times 3$ before activation. We also see that, due to the Maxwell stress, the electrode areas own smaller mechanical stress than the passive areas. Fig. 8(f) shows the distribution of the electric fields within the electrode areas. Larger electric fields occur within regions that are closer to the passive areas, which agrees well with the nominal strain distribution along the thickness direction as shown in Fig. 8(c).

\section{EXPERIMENTS}

In this section, we will show the experimental results of the three cases in Section $\mathrm{V}$ to experimentally validate the effectiveness of our design methodology. Their intuitive design counterparts are also evaluated for comparison.

\section{A. Setup}

The experimental setup is shown in Fig. 9. The fabricated DEA is connected to the high-voltage amplifier (10/10B-HS, Trek, Inc.) and is controlled by a dSPACE-DS1103 control board equipped with digital-to-analog converters (DACs). The DACs are used to output analog ramping voltages that is amplified by the high voltage amplifier with a fixed gain of 1000 . The ramping rate is set to be $100 \mathrm{~V} / \mathrm{s}$. The MAT$\mathrm{LAB} /$ Simulink software is used to implement the algorithms, which are directly downloaded to the dSPACE-DS1103 control board via the ControlDesk interface. The real-time states of the DEAs are captured by a camera and through image processing the induced displacements can be obtained.

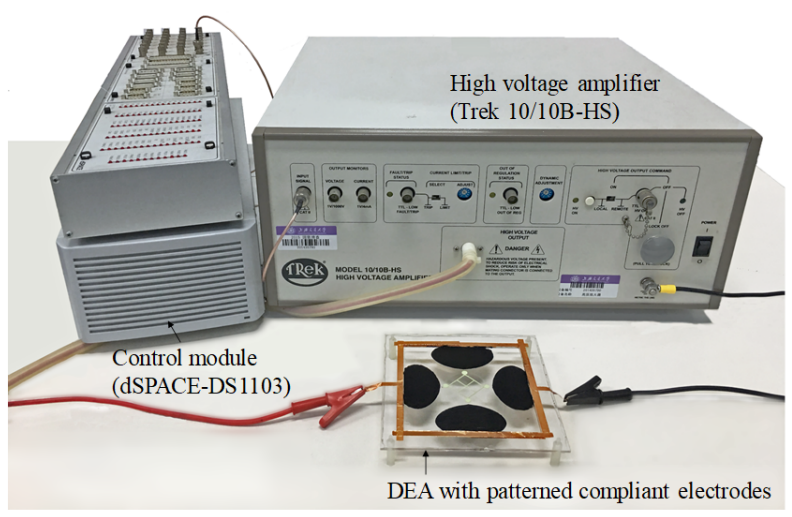

Fig. 9: The experimental setup.

\section{B. Fabrication of DEAs}

The DEAs were fabricated as follows: i) biaxially stretched a piece of VHB 4910 sheet by $3 \times 3$ with a custom-built stretcher; ii) a stiff acrylic frame (thickness, $3 \mathrm{~mm}$ ) fabricated by a laser cutting machine (VLS3.50-SYS) was utilized to take the prestretched dielectric elastomer membrane down from the stretcher and support the membrane; iii) based on the optimized SEF profile, a release paper based mask was fabricated by the laser cutting machine and then attached onto both sides of the VHB; iv) coated the electrodes (carbon grease) with a paintbrush on both sides of the SEF area; v) removed the mask and connected the electrodes to the amplifier with copper tapes. 


\section{Results}

For the three cases each, we apply one high-voltage power to both the optimal DEA and its intuitive design counterpart so that they are always supplied with the same voltage. The experiments are performed in triplicate for each case and the average displacements are adopted for evaluation. Fig. 10 shows the experimental results. For convenience of description, we denote the concerned displacement magnitudes of the optimal design and the intuitive design by $J^{\text {opt }}$ and $J^{\text {non }}$, respectively, and define the relative improvement of the optimized design by

$$
\alpha=\frac{J^{\text {opt }}-J^{\text {non }}}{J^{\text {non }}} \times 100 \% .
$$

In particular, for Case III, we adopt the average displacement of the four points for evaluation. It is observed that, for all the three cases, the optimized designs remarkably outperform their intuitive counterparts, resulting in relative improvements of $18.89 \%, 34.18 \%$ and $25.28 \%$ at $6 \mathrm{kV}$, as shown in Fig. 10.

We further investigate the robustness of the optimized designs by evaluating their performances on the whole voltage range, i.e. from $0 \mathrm{kV}$ until electric breakdown around $8 \mathrm{kV}$. As shown in Fig. 10, the superiority of the optimized designs grows pronounced when the applied voltage increases beyond $6 \mathrm{kV}$, achieving relative improvements of $48.51 \%, 41.14 \%$ and $76.47 \%$ for the three cases. This robustness is significant and advantageous for practical applications in which the applied voltage typically spans quite large ranges.

Remark 7. It is well known that DEAs may suffer material failures such as mechanical rupture, electric breakdown, electromechanical instability or loss of tension with high voltages. Electromechanical instability is not included in the optimization framework due to the induced discontinuity, and it is well suppressed by the prestretch. When the applied voltage approaches $8 \mathrm{kV}$, the DEA membranes are prone to loss of tension and electric breakdown. It is noted that, the critical electric field for breakdown depends on the stretches the DEA undergoes [43]. To incorporate electric breakdown into the design optimization framework, it requires imposing a constraint on the stretch along the thickness direction.

\section{APPLICATIONS}

DEAs may find various applications by interacting with host structures, to realize novel soft machines and robots. Shapemorphing structures are at the core of future applications in smart materials and structures [44]-[47] and robots [48][50]. In contrast to existing mechanisms for creating shape changes, e.g. mechanical stretches [44], pressurization [46] and temperature loading [47], the configurational changes driven by DEAs are faster and amenable to electrical control and programing.

We aim to drive thin-walled host structures to shape-morph from flat sheets to desired three dimensional configurations by inducing buckling. Starting from this high-level motion task, our design method automatically produces the optimal SEF (as shown in Case III) that equips the DEA with large motion range to enable shape-morphing of various structures. The host structures made of hard paper (with thickness of $50 \mu \mathrm{m}$ ) have different shapes at rest, as shown by their two dimensional precursors in Fig. 11. The feet of the host structures (highlighted by the red areas) are attached to the DEA (note that VHB 4910 is highly adhesive), while their bodies (highlighted by the blue areas) are smeared with silicone oil and thus are able to detach themselves from the DEA.

For the four host structures each, we increase the applied voltage gradually from $0 \mathrm{kV}$ to $7.5 \mathrm{kV}$, without encountering any material failures. It is observed that each host structure would start to buckle at a certain voltage, which can be defined as the critical buckling voltage. Specifically, the critical buckling voltages for the four host structures are $5.0 \mathrm{kV}$, $5.4 \mathrm{kV}, 5.3 \mathrm{kV}$ and $4.8 \mathrm{kV}$. The initial and final deformed configurations are shown in Fig. 11 and the dynamic configurational transitions are shown in the supplemental video S4. The simulation results conducted in ABAQUS well predict the deformed configurations.

We may envisage many more potential applications with the optimized DEAs. For example, the current DEA prototype can be directly extended to planar positioning systems with enlarged workspace of the end-effector. Further, a variety of actuator configurations of DEAs such as minimum energy structures [51], [52] and bulged membranes [28] that have been applied to drive robots [24], [25], haptic actuators [26], fluid pumps [53], etc, can benefit from the improved deformability offered directly by optimizing SEFs without the need for additional cumbersome mechanical design.

\section{DISCUSSION AND CONCLUSION}

In this paper, we propose a computational design methodology for DEAs to systematically explore their actuation capabilities, by leveraging the freeform electrode patterns. Our approach in theory allows the electrodes to take amorphous geometric profile without prior knowledge, and searches for the optimal design by a gradient-based optimizer. We address the challenge of embedding the highly nonlinear behavior of DEAs into the optimization model. We validate the effectiveness of our method by automatically creating the optimal SEFs for DEAs targeting at various motion tasks. We show that the optimized DEAs can be used to trigger shape-morphing of various structures, among many other potential applications.

We note that researchers have also explored the use of evolutionary algorithms, e.g. genetic algorithms, to aid in the design process of DEAs [54], [55], in which the design variable, i.e. the population, is updated by combining or mutating existing designs in a stochastic fashion. However, genetic algorithms are essentially randomized heuristic search strategies with poor scalability, and typically result in premature convergence and slow convergent speed. The present design problems of DEAs can be cast as continuous optimization problems, and as demonstrated, closed-form derivatives can be obtained. Therefore, we adopt a gradient-based topology optimization approach to provide a systematic exploration of the freeform design space of SEFs.

In a general sense, our approach paves the way for shifting the design of soft actuators and robots from classical intuitive 


\section{(a) Case I}

\begin{tabular}{ccccccc}
\hline Voltage $(\mathrm{kV})$ & 4 & 5 & 6 & 7 & 7.5 & 8 \\
\hline$\alpha$ & $20.74 \%$ & $16.60 \%$ & $18.89 \%$ & $24.09 \%$ & $25.76 \%$ & $48.51 \%$
\end{tabular}

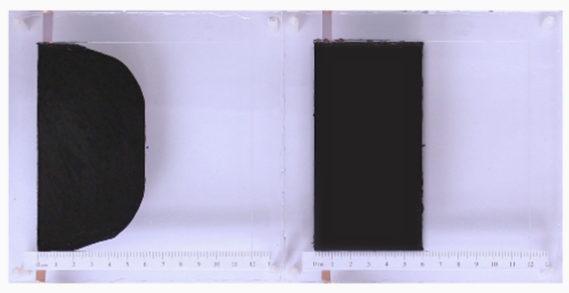

$\Phi=0 \mathrm{kV}$

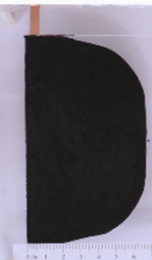

$$
\Phi=6 \mathrm{kV}
$$
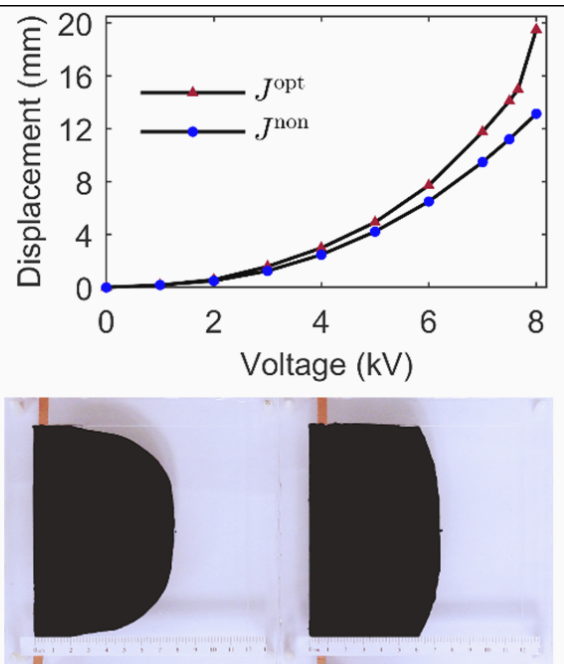

$\Phi=8 \mathrm{kV}$

(b) Case II

\begin{tabular}{ccccccc}
\hline Voltage $(\mathrm{kV})$ & 4 & 5 & 6 & 7 & 7.5 & 7.8 \\
\hline$\alpha$ & $31.21 \%$ & $30.29 \%$ & $34.18 \%$ & $35.73 \%$ & $40.43 \%$ & $41.14 \%$ \\
\hline
\end{tabular}

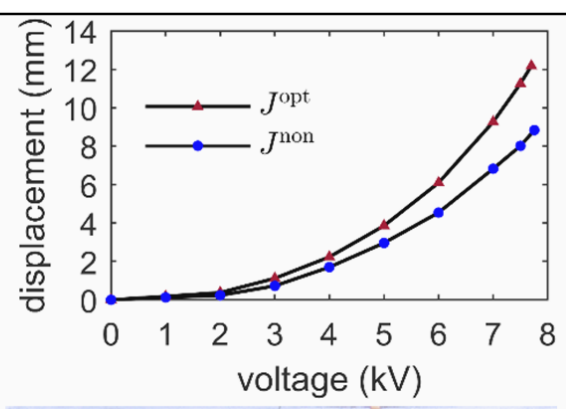

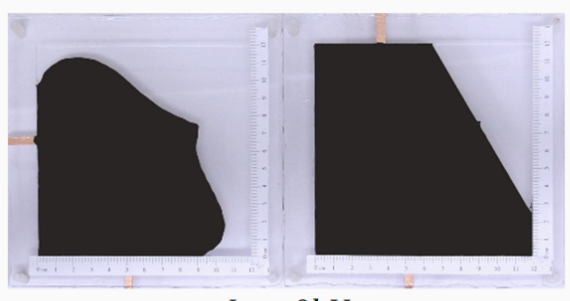

$\Phi=0 \mathrm{kV}$

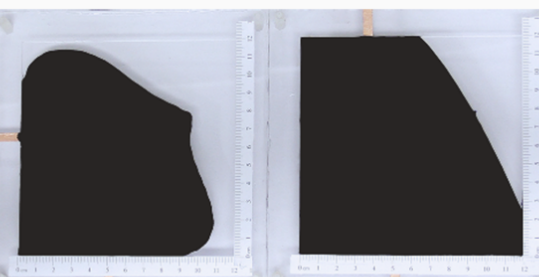

$\Phi=6 \mathrm{kV}$

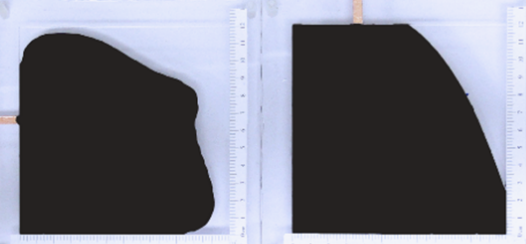

$\Phi=7.8 \mathrm{kV}$

(c) Case III

\begin{tabular}{ccccccc}
\hline Voltage $(\mathrm{kV})$ & 4 & 5 & 6 & 7 & 7.5 & 8 \\
\hline$\alpha$ & $0.26 \%$ & $12.55 \%$ & $25.28 \%$ & $37.42 \%$ & $39.02 \%$ & $76.47 \%$ \\
\hline
\end{tabular}
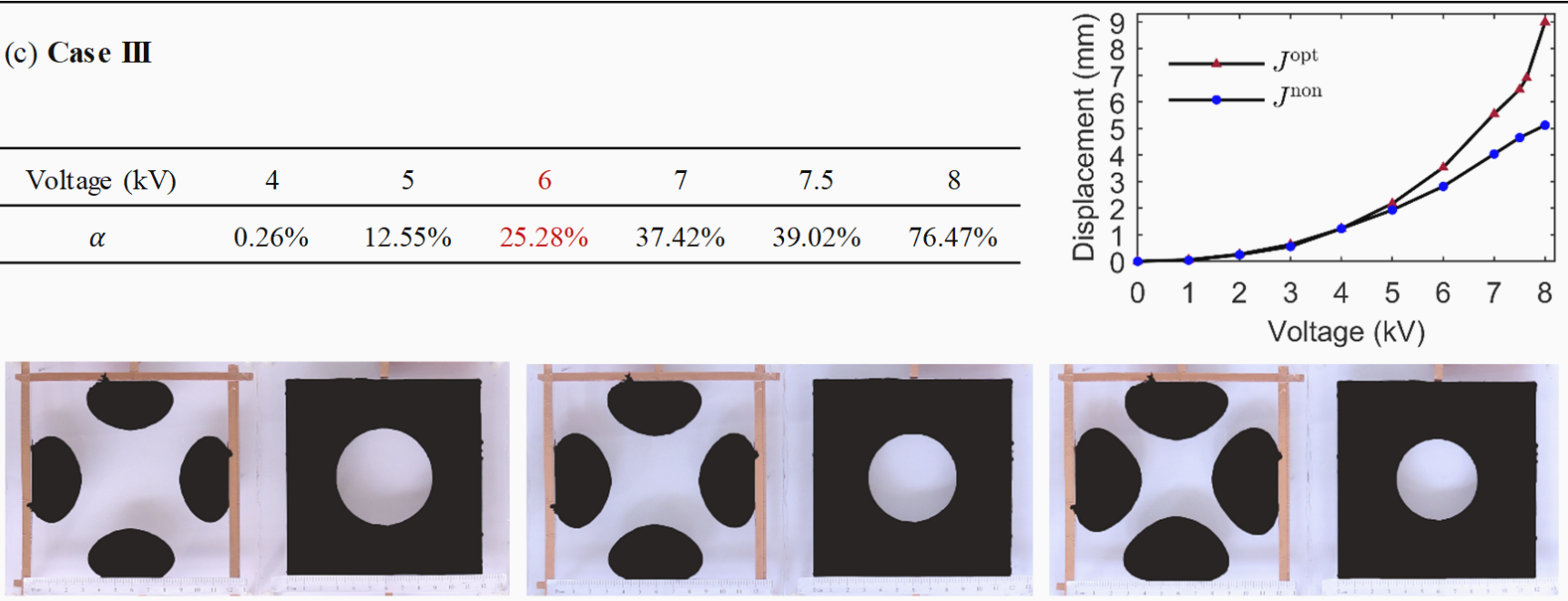

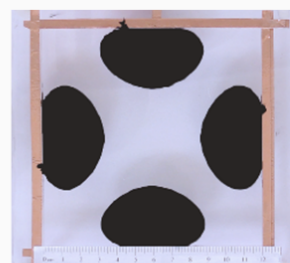

$\Phi=6 \mathrm{kV}$

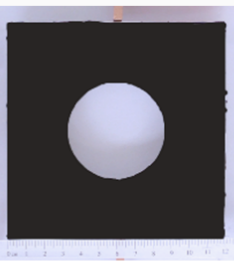

$\Phi=0 \mathrm{kV}$
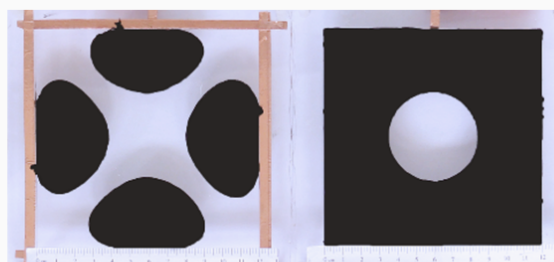

$\Phi=8 \mathrm{kV}$

Fig. 10: The experimental results of the three cases. 


\begin{tabular}{|l|l|l|l|c|}
\hline 2D precursor & Simulation (FEA) & Experiment $(\Phi=0 \mathrm{kV})$ & Experiment $(\Phi=7.5 \mathrm{kV})$ & $\begin{array}{c}\text { Critical buckling } \\
\text { voltage }(\mathrm{kV})\end{array}$ \\
\hline & & & & 5.0 \\
\hline
\end{tabular}

Fig. 11: The applications of optimized DEAs for triggering buckling of host structures from planar sheets to desired three dimensional configurations.

or empirical design paradigms to a more systematic process based on mathematics and optimization theories. With the unconstrained design space in terms of the geometric configuration [56], [57], material distributions [58] and volumetric actuations [59], enabled by additive manufacturing techniques and fast simulation algorithms [4], [60], design optimization approaches are becoming increasingly important and indispensable to generate novel mechanisms and deformable robots.

In the future, we hope to extend the proposed optimization algorithm to DEAs of different configurations and more complex motion specifications for which intuitive designs are extremely hard to apply, e.g. prestretched DEAs coated on planar precursors to form three-dimensional dielectric elastomer minimum energy structures (DEMES). DEMES typically involve buckling process of the coupled system consisting of DEAs and compliant frames, into a state of minimum energy. The configurations of DEAs mathematically correspond to the induced buckling modes. Therefore, design optimization of DEMES will pose great challenge in finite element electromechanical analysis and the sensitivity analysis. Nevertheless, the overall performance of DEMES can benefit from the concurrent optimization with respect to the frame shape and the SEF. On activating the spatiotemporally-varying electric fields, the DEMES may undergo desired configurational transformation to perform complex tasks. We envisage that systematic design optimization can uncover untapped potential of DEAs and propel their applications in soft robots through improved deformability.

\section{APPENDIX A}

Nominal Stress AND MATERIAL TENSOR

The nominal stress, also known as the first Piola-Kirchhoff stress, takes the following form

$$
\begin{aligned}
& s_{i j}=\frac{\partial W}{\partial F_{i j}} \\
& =\mu\left(\frac{1}{\bar{J}^{2 / 3}} F_{i j}-\frac{1}{3} \bar{I}_{1} F_{j i}^{-1}\right)+\kappa \bar{J}(\bar{J}-1) F_{j i}^{-1} \\
& -\frac{1}{2} \varepsilon \bar{J} \tilde{E}_{p} \tilde{E}_{q}\left(F_{p k}^{-1} F_{q k}^{-1} F_{j i}^{-1}-F_{p i}^{-1} F_{j k}^{-1} F_{q k}^{-1}-F_{p k}^{-1} F_{q i}^{-1} F_{j k}^{-1}\right)
\end{aligned}
$$

For a planar DEA where the electric field is applied along the thickness direction, the nominal stress reduces to

$$
\begin{aligned}
s_{i j}= & \mu\left(\frac{1}{\bar{J}^{2 / 3}} F_{i j}-\frac{1}{3} \bar{I}_{1} F_{j i}^{-1}\right)+\kappa \bar{J}(\bar{J}-1) F_{j i}^{-1} \\
& -\frac{1}{2} \varepsilon \bar{J} \tilde{E}^{2} F_{33}^{-2}\left(F_{j i}^{-1}-2 F_{33}^{-1} \delta_{i 3} \delta_{j 3}\right)
\end{aligned}
$$

with the mechanical and electric components stated as follows

$$
\begin{gathered}
s_{i j}^{u}=\mu\left(\frac{1}{\bar{J}^{2 / 3}} F_{i j}-\frac{1}{3} \bar{I}_{1} F_{j i}^{-1}\right)+\kappa \bar{J}(\bar{J}-1) F_{j i}^{-1}, \\
s_{i j}^{e}=-\frac{1}{2} \varepsilon \bar{J} \tilde{E}^{2} F_{33}^{-2}\left(F_{j i}^{-1}-2 F_{33}^{-1} \delta_{i 3} \delta_{j 3}\right) .
\end{gathered}
$$


The material tensor is derived by differentiating the nominal stress with respect to the deformation gradient, i.e. $\mathbf{C}=$ $\partial \mathbf{s} / \partial \mathbf{F}$, and its tensor form is expressed by

$$
\begin{aligned}
C_{i j k l}= & \frac{\partial s_{i j}}{\partial F_{k l}} \\
= & \frac{\mu}{\bar{J}^{2 / 3}}\left(-\frac{2}{3} F_{l k}^{-1} F_{i j}+\delta_{i k} \delta_{j l}-\frac{2}{3} F_{k l} F_{j i}^{-1}\right) \\
& +\left[\frac{2}{9} \mu \bar{I}_{1}+\kappa \bar{J}(2 \bar{J}-1)-\frac{1}{2} \varepsilon \bar{J} \tilde{E}^{2} F_{33}^{-2}\right] F_{l k}^{-1} F_{j i}^{-1} \\
& +\left[\frac{1}{3} \mu \bar{I}_{1}-\kappa \bar{J}(\bar{J}-1)+\frac{1}{2} \varepsilon \bar{J} \tilde{E}^{2} F_{33}^{-2}\right] F_{j k}^{-1} F_{l i}^{-1}
\end{aligned}
$$

\section{APPENDIX B \\ Adjoint SEnsitivity AnALYsis}

The material derivative of the Lagrangian with respect to a pseudo-time $\tau$, i.e. $\dot{L}=\mathrm{d} L / \mathrm{d} \tau$, is given by,

$$
\dot{L}=\dot{J}(\mathbf{u})+\dot{a}(\mathbf{u}, \mathbf{w}, \Omega)+\lambda \dot{\varsigma} .
$$

The material derivative of the deformation gradient is stated by

$$
\dot{\mathbf{F}}=\nabla_{0} \dot{\mathbf{u}}-\nabla_{0} \mathbf{u} \nabla_{0} \mathbf{V}
$$

According to Lemma 6.1. in [61], the material derivatives $\dot{a}(\mathbf{u}, \mathbf{w}, \Omega)$ and $\dot{\varsigma} \mathrm{read}$,

$$
\begin{gathered}
\dot{a}(\mathbf{u}, \mathbf{w}, \Omega) \\
=\int_{\Omega}\left[\dot{\mathbf{s}}: \nabla_{0} \mathbf{w}+\mathbf{s}:\left(\nabla_{0} \mathbf{w}\right)^{\cdot}+\left(\mathbf{s}: \nabla_{0} \mathbf{w}\right) \nabla_{0} \cdot \mathbf{V}\right] \mathrm{d} \Omega \\
=\int_{\Omega}\left[\mathbf{C}:\left(\nabla_{0} \dot{\mathbf{u}}-\nabla_{0} \mathbf{u} \nabla_{0} \mathbf{V}\right): \nabla_{0} \mathbf{w}+\right. \\
\left.\mathbf{s}:\left(\nabla_{0} \dot{\mathbf{w}}-\nabla_{0} \mathbf{w} \nabla_{0} \mathbf{V}\right)+\left(\mathbf{s}: \nabla_{0} \mathbf{w}\right) \nabla_{0} \cdot \mathbf{V}\right] \mathrm{d} \Omega \\
\dot{\varsigma}=\int_{\Omega} \nabla_{0} \cdot \mathbf{V} \mathrm{d} \Omega .
\end{gathered}
$$

The adjoint equation is established by collecting all the terms related to $\dot{\mathbf{u}}$ in (28) and making their sum zero, which yields,

$$
\frac{\mathrm{d} J(\mathbf{u})}{\mathrm{d} \mathbf{u}} \dot{\mathbf{u}}+\int_{\Omega} \mathbf{C}: \nabla_{0} \dot{\mathbf{u}}: \nabla_{0} \mathbf{w} \mathrm{d} \Omega=0 .
$$

Since the design objective is expressed in term of $\mathbf{u}$, its derivative with respect to $\mathbf{u}$ can be easily evaluated during numerical implementation. To ensure (32) holds for any $\dot{\mathbf{u}}$ necessitates a unique $\mathbf{w}$, i.e. the so-called adjoint displacement. Numerically, we discretize (32), extract the nodal displacement derivative $\dot{\mathbf{u}}$ as a common factor, and then directly obtain the adjoint displacement $\mathbf{w}$ by solving the linear equation. Thereafter, the material derivative of the Lagrangian can be readily obtained [see (16)].

\section{APPENDIX C \\ Design Velocity Selection}

A 4-node rectangular grid is adopted to discretize the design domain. A continuum displacement field within the eth element can be interpolated by,

$$
\mathbf{u}(\mathbf{X})=\left[\begin{array}{cc}
N^{1}(\mathbf{X}) & 0 \\
0 & N^{1}(\mathbf{X}) \\
N^{2}(\mathbf{X}) & 0 \\
0 & N^{2}(\mathbf{X}) \\
N^{3}(\mathbf{X}) & 0 \\
0 & N^{3}(\mathbf{X}) \\
N^{4}(\mathbf{X}) & 0 \\
0 & N^{4}(\mathbf{X})
\end{array}\right]^{\mathrm{T}} \quad \mathbf{u}^{e}, \quad \forall \mathbf{X} \in \Omega_{e}
$$

where $\mathbf{u}^{e}=\left[\begin{array}{llllllll}u_{x}^{1} & u_{y}^{1} & u_{x}^{2} & u_{y}^{2} & u_{x}^{3} & u_{y}^{3} & u_{x}^{4} & u_{y}^{4}\end{array}\right]^{\mathrm{T}}$ denotes the nodal displacement vector in the $e$ th element, $\Omega_{e}$ denotes the domain of the eth element, $N^{i}(\mathbf{X})$ is the bilinear interpolation function, the superscripts $i=1,2,3,4$ denote the local nodal indices, and the subscripts $x$ and $y$ correspond to the axial directions in the Cartesian coordinate system. Similarly, the adjoint displacement field $\mathbf{w}$, the derivative of the displacement field $\dot{\mathbf{u}}$ and the design velocities $\mathbf{V}$ within the $e$ th element are also interpolated in the same form as in (33), whose nodal component vectors are denoted by $\mathbf{w}^{e}, \dot{\mathbf{u}}^{e}$ and $\mathbf{V}^{e}$, respectively.

The gradient of the displacement field can be stated by

$$
\nabla_{0} \mathbf{u}(\mathbf{X})=\left[\begin{array}{llll}
u_{x}^{1} & u_{x}^{2} & u_{x}^{3} & u_{x}^{4} \\
u_{y}^{1} & u_{y}^{2} & u_{y}^{3} & u_{y}^{4}
\end{array}\right]\left[\begin{array}{cc}
N_{, x}^{1} & N_{, y}^{1} \\
N_{, x}^{2} & N_{, y}^{2} \\
N_{, x}^{3} & N_{, y}^{3} \\
N_{, x}^{4} & N_{, y}^{4}
\end{array}\right]
$$

with $N_{, x}^{i}=\partial N^{i}(\mathbf{X}) / \partial x, N_{, y}^{i}=\partial N^{i}(\mathbf{X}) / \partial y$. To extract the nodal components $\mathbf{u}^{e}$, we define a vectorization process $\mapsto$ : $\nabla_{0} \mathbf{u} \mapsto \mathbf{u}_{\nabla}=\left[\begin{array}{llll}u_{11} & u_{21} & u_{12} & u_{22}\end{array}\right]^{\mathrm{T}}=\mathrm{Hu}^{e}$, with $\mathbf{H}$ taking the following form,

$$
\mathbf{H}=\left[\begin{array}{cccccccc}
N_{, x}^{1} & 0 & N_{, x}^{2} & 0 & N_{, x}^{3} & 0 & N_{, x}^{4} & 0 \\
0 & N_{, x}^{1} & 0 & N_{, x}^{2} & 0 & N_{, x}^{3} & 0 & N_{, x}^{4} \\
N_{, y}^{1} & 0 & N_{, y}^{2} & 0 & N_{, y}^{3} & 0 & N_{, y}^{4} & 0 \\
0 & N_{, y}^{1} & 0 & N_{, y}^{2} & 0 & N_{, y}^{3} & 0 & N_{, y}^{4}
\end{array}\right] .
$$

Similarly, the gradients of other terms are vectorized by $\nabla_{0} \dot{\mathbf{u}} \mapsto \dot{\mathbf{u}}_{\nabla}=\mathbf{H} \dot{\mathbf{u}}^{e}, \nabla_{0} \mathbf{w} \mapsto \mathbf{w}_{\nabla}=\mathbf{H w}^{e}$ and $\nabla_{0} \mathbf{V} \mapsto \mathbf{V}_{\nabla}=\mathbf{H} \mathbf{V}^{e}$. Consequently, the matrix multiplication $\nabla_{0} \mathbf{u} \nabla_{0} \mathbf{V}$ can be evaluated at the nodes,

$$
\nabla_{0} \mathbf{u} \nabla_{0} \mathbf{V} \mapsto \mathbf{V}_{\nabla \mathbf{u} \nabla}=\underbrace{\left[\begin{array}{llll}
u_{11} & u_{12} & \\
u_{21} & u_{22} & \\
& & u_{11} & u_{12} \\
& & u_{21} & u_{22}
\end{array}\right]}_{\tilde{\mathbf{u}}} \underbrace{\left[\begin{array}{c}
V_{11} \\
V_{21} \\
V_{12} \\
V_{22}
\end{array}\right]}_{\mathbf{V}_{\nabla}}
$$

where $\tilde{\mathbf{u}}=\operatorname{diag}\left(\nabla_{0} \mathbf{u}, \nabla_{0} \mathbf{u}\right)$, and similarly, we may vectorize $\nabla_{0} \mathbf{w} \nabla_{0} \mathbf{V}$ to be $\tilde{\mathbf{w}} \mathbf{H} \mathbf{V}^{e}$ with $\tilde{\mathbf{w}}=\operatorname{diag}\left(\nabla_{0} \mathbf{w}, \nabla_{0} \mathbf{w}\right)$.

The divergence of the velocity can be stated by,

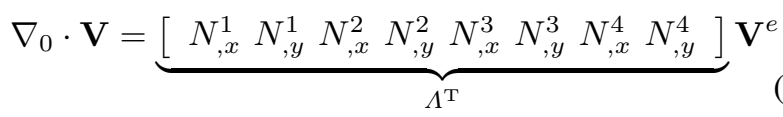


It should be noted that only the normal velocity contributes to the evolution process. To convert the nodal components $\mathbf{V}^{e}$ to the normal velocities $\mathbf{V}_{n}^{e}$, it has,

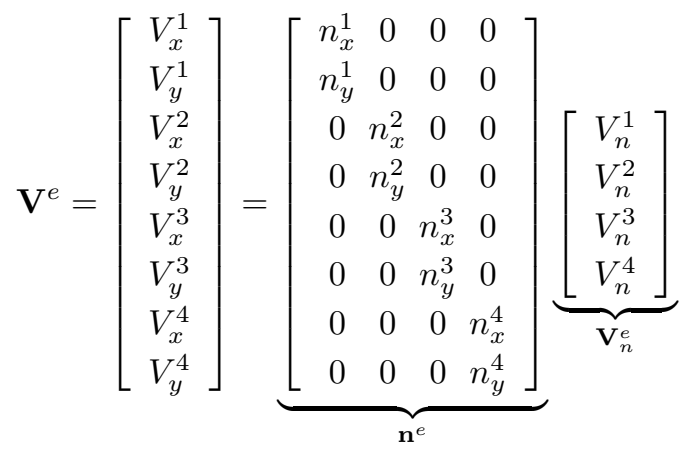

where $\mathbf{n}^{e}$ denotes the transformation matrix containing the components of nodal normal vectors within the element, in the direction of decreasing $\phi$.

With vectorized terms above, we can discretize the material derivative of the Lagrangian in (16). The first integral term is rewritten by,

$$
\mathbf{C}:\left(\nabla_{0} \mathbf{u} \nabla_{0} \mathbf{V}\right): \nabla_{0} \mathbf{w}=\mathbf{w}_{\nabla}^{\mathrm{T}}\left(\mathbf{C}^{*}\right)^{\mathrm{T}} \tilde{\mathbf{u}} \mathbf{H} \mathbf{V}^{e}
$$

where the nominal stress tensor $\mathbf{s}$ is also vectorized by $\mathbf{s} \mapsto$ $\mathbf{s}_{V}=\left[\begin{array}{llll}s_{11} & s_{21} & s_{12} & s_{22}\end{array}\right]^{\mathrm{T}}$, and the fourth-order material tensor is written in matrix form, denoted by $\mathbf{C}^{*}$

$$
\mathbf{C}^{*}=\left[\begin{array}{llll}
C_{1111} & C_{2111} & C_{1211} & C_{2211} \\
C_{1121} & C_{2121} & C_{1221} & C_{221} \\
C_{1112} & C_{2112} & C_{1212} & C_{2212} \\
C_{1122} & C_{2122} & C_{1222} & C_{222}
\end{array}\right] .
$$

The second integral term is rewritten by

$$
\mathbf{s}: \nabla_{0} \mathbf{w} \nabla_{0} \mathbf{V}=\mathbf{s}_{V}^{\mathrm{T}} \tilde{\mathbf{w}} \mathbf{H} \mathbf{V}^{e} .
$$

The last integral term is rewritten by

$$
\left(\mathbf{s}: \nabla_{0} \mathbf{w}+\lambda\right) \nabla_{0} \cdot \mathbf{V}=\left(\mathbf{s}_{V}^{\mathrm{T}} \mathbf{w}_{\nabla}+\lambda\right) \Lambda^{\mathrm{T}} \mathbf{V}^{e} .
$$

By substituting all these results into (16), it yields,

$$
\begin{aligned}
\dot{L}=\left\{\begin{array}{l}
N_{e} \\
\stackrel{A}{e=1}
\end{array} \int_{{ }_{0} \Omega^{e}}[\right. & -\mathbf{w}_{\nabla}^{\mathrm{T}}\left(\mathbf{C}^{*}\right)^{\mathrm{T}} \tilde{\mathbf{u}} \mathbf{H}-\mathbf{s}_{V}^{\mathrm{T}} \tilde{\mathbf{w}} \mathbf{H} \\
& \left.\left.+\left(\mathbf{s}_{V}^{\mathrm{T}} \mathbf{w}_{\nabla}+\lambda\right) \Lambda^{\mathrm{T}}\right] \mathbf{n}^{e} \mathrm{~d} \Omega\right\} \mathbf{V}_{n}
\end{aligned}
$$

where $N_{e}$ denotes the number of elements and $\mathbf{V}_{n}$ is the collection vector of the normal velocities for the grid points. Note that the Lagrangian multiplier in (43) is only active in the elements activated by the SEF. To this end, for ensuring the steepest descent direction, the normal velocities of the grid points can be selected [see (17)].

\section{ACKNOWLEDGMENT}

The authors would like to appreciate insightful discussion with Professor Michael Yu Wang, Professor Yangjun Luo and Dr. Hareesh Godaba.

\section{REFERENCES}

[1] D. Rus and M. T. Tolley, "Design, fabrication and control of soft robots," Nature, vol. 521, no. 7553, pp. 467-475, 2015.

[2] S. Yim and M. Sitti, "Shape-programmable soft capsule robots for semiimplantable drug delivery," IEEE Transactions on Robotics, vol. 28, no. 5, pp. 1198-1202, 2012.

[3] Y. Kim, H. Yuk, R. Zhao, S. A. Chester, and X. Zhao, "Printing ferromagnetic domains for untethered fast-transforming soft materials," Nature, vol. 558, no. 7709, p. 274, 2018.

[4] O. Goury and C. Duriez, "Fast, generic, and reliable control and simulation of soft robots using model order reduction," IEEE Transactions on Robotics, no. 99, pp. 1-12, 2018.

[5] J. Yi, X. Chen, C. Song, J. Zhou, Y. Liu, S. Liu, and Z. Wang, "Customizable three-dimensional-printed origami soft robotic joint with effective behavior shaping for safe interactions," IEEE Transactions on Robotics, vol. 35, no. 1, pp. 114-123, 2019.

[6] B. Mosadegh, P. Polygerinos, C. Keplinger, S. Wennstedt, R. F. Shepherd, U. Gupta, J. Shim, K. Bertoldi, C. J. Walsh, and G. M. Whitesides, "Pneumatic networks for soft robotics that actuate rapidly," Advanced Functional Materials, vol. 24, no. 15, pp. 2163-2170, 2014.

[7] J. Bishop-Moser and S. Kota, "Design and modeling of generalized fiberreinforced pneumatic soft actuators," IEEE Transactions on Robotics, vol. 31, no. 3, pp. 536-545, 2015.

[8] P. Polygerinos, Z. Wang, J. T. Overvelde, K. C. Galloway, R. J. Wood, K. Bertoldi, and C. J. Walsh, "Modeling of soft fiber-reinforced bending actuators," IEEE Transactions on Robotics, vol. 31, no. 3, pp. 778-789, 2015.

[9] A. D. Marchese and D. Rus, "Design, kinematics, and control of a soft spatial fluidic elastomer manipulator," The International Journal of Robotics Research, vol. 35, no. 7, pp. 840-869, 2016.

[10] Y. Li, Y. Chen, Y. Yang, and Y. Wei, "Passive particle jamming and its stiffening of soft robotic grippers," IEEE Transactions on Robotics, vol. 33, no. 2, pp. 446-455, 2017.

[11] H. Rodrigue, W. Wang, M.-W. Han, T. J. Kim, and S.-H. Ahn, "An overview of shape memory alloy-coupled actuators and robots," Soft robotics, vol. 4, no. 1, pp. 3-15, 2017.

[12] A. Firouzeh, M. Salerno, and J. Paik, "Stiffness control with shape memory polymer in underactuated robotic origamis," IEEE Transactions on Robotics, vol. 33, no. 4, pp. 765-777, 2017.

[13] R. Pelrine, R. Kornbluh, Q. Pei, and J. Joseph, "High-speed electrically actuated elastomers with strain greater than $100 \%$," Science, vol. 287 , no. 5454, pp. 836-839, 2000

[14] F. Carpi, S. Bauer, and D. De Rossi, "Stretching dielectric elastomer performance," Science, vol. 330, no. 6012, pp. 1759-1761, 2010.

[15] G.-Y. Gu, J. Zhu, L.-M. Zhu, and X. Zhu, "A survey on dielectric elastomer actuators for soft robots," Bioinspiration \& biomimetics, vol. 12, no. 1, p. 011003, 2017.

[16] G.-Y. Gu, U. Gupta, J. Zhu, L.-M. Zhu, and X. Zhu, "Modeling of viscoelastic electromechanical behavior in a soft dielectric elastomer actuator," IEEE Transactions on Robotics, vol. 33, no. 5, pp. 1263-1271, 2017.

[17] J. Zou and G. Gu, "High-precision tracking control of a soft dielectric elastomer actuator with inverse viscoelastic hysteresis compensation," IEEE/ASME Transactions on Mechatronics, vol. 24, no. 1, pp. 36-44, 2019.

[18] J. Shintake, S. Rosset, B. Schubert, D. Floreano, and H. Shea, "Versatile soft grippers with intrinsic electroadhesion based on multifunctional polymer actuators," Advanced Materials, vol. 28, no. 2, pp. 231-238, 2016.

[19] G.-K. Lau, K.-R. Heng, A. S. Ahmed, and M. Shrestha, "Dielectric elastomer fingers for versatile grasping and nimble pinching," Applied Physics Letters, vol. 110, no. 18, p. 182906, 2017.

[20] S. Pourazadi, H. T. Bui, and C. Menon, "Investigation on a soft grasping gripper based on dielectric elastomer actuators," Smart Materials and Structures, vol. 28, no. 3, 2019.

[21] H. Godaba, J. Li, Y. Wang, and J. Zhu, "A soft jellyfish robot driven by a dielectric elastomer actuator," IEEE Robotics and Automation Letters, vol. 1, no. 2, pp. 624-631, 2016.

[22] T. Li, G. Li, Y. Liang, T. Cheng, J. Dai, X. Yang, B. Liu, Z. Zeng, Z. Huang, Y. Luo et al., "Fast-moving soft electronic fish," Science Advances, vol. 3, no. 4, p. e1602045, 2017.

[23] C. Christianson, N. N. Goldberg, D. D. Deheyn, S. Cai, and M. T. Tolley, "Translucent soft robots driven by frameless fluid electrode dielectric elastomer actuators," Science Robotics, vol. 3, no. 17, p. eaat1893, 2018. 
[24] J. Cao, L. Qin, J. Liu, Q. Ren, C. C. Foo, H. Wang, H. P. Lee, and J. Zhu, "Untethered soft robot capable of stable locomotion using soft electrostatic actuators," Extreme Mechanics Letters, vol. 21, pp. 9-16, 2018.

[25] G. Gu, J. Zou, R. Zhao, X. Zhao, and X. Zhu, "Soft wall-climbing robots," Science Robotics, vol. 3, no. 25, p. eaat2874, 2018.

[26] I. M. Koo, K. Jung, J. C. Koo, J.-D. Nam, Y. K. Lee, and H. R. Choi, "Development of soft-actuator-based wearable tactile display," IEEE Transactions on Robotics, vol. 24, no. 3, pp. 549-558, 2008.

[27] P. Lotz, M. Matysek, and H. F. Schlaak, "Fabrication and application of miniaturized dielectric elastomer stack actuators," Mechatronics, IEEE/ASME Transactions on, vol. 16, no. 1, pp. 58-66, 2011.

[28] F. Chen, J. Cao, H. Zhang, M. Y. Wang, J. Zhu, and Y. Zhang, "Programmable deformations of networked inflated dielectric elastomer actuators," IEEE/ASME Transactions on Mechatronics, vol. 24, no. 1, pp. 45-55, 2019.

[29] S. Mintchev, J. Shintake, and D. Floreano, "Bioinspired dual-stiffness origami," Science Robotics, vol. 3, no. 20, p. eaau0275, 2018.

[30] A. Poulin, M. Imboden, F. Sorba, S. Grazioli, C. Martin-Olmos, S. Rosset, and $\mathrm{H}$. Shea, "An ultra-fast mechanically active cell culture substrate," Scientific reports, vol. 8, no. 1, p. 9895, 2018.

[31] L. Calabrese, G. Frediani, M. Gei, D. De Rossi, and F. Carpi, "Active compression bandage made of electroactive elastomers," IEEE/ASME Transactions on Mechatronics, vol. 23, no. 5, pp. 2328-2337, 2018.

[32] Z. Suo, "Theory of dielectric elastomers," Acta Mechanica Solida Sinica, vol. 23, no. 6, pp. 549-578, 2010.

[33] Z. Zou, T. Li, S. Qu, and H. Yu, "Active shape control and phase coexistence of dielectric elastomer membrane with patterned electrodes," Journal of Applied Mechanics, vol. 81, no. 3, p. 031016, 2014.

[34] C. T. Nguyen, H. Phung, P. T. Hoang, T. D. Nguyen, H. Jung, and H. R. Choi, "Development of an insect-inspired hexapod robot actuated by soft actuators," Journal of Mechanisms and Robotics, vol. 10, no. 6, p. 061016, 2018.

[35] E. Hajiesmaili and D. R. Clarke, "Reconfigurable shape-morphing dielectric elastomers using spatially varying electric fields," Nature communications, vol. 10, no. 1, p. 183, 2019.

[36] S. Osher and J. A. Sethian, "Fronts propagating with curvaturedependent speed: algorithms based on hamilton-jacobi formulations," Journal of computational physics, vol. 79, no. 1, pp. 12-49, 1988.

[37] M. Y. Wang, X. Wang, and D. Guo, "A level set method for structural topology optimization," Computer methods in applied mechanics and engineering, vol. 192, no. 1, pp. 227-246, 2003.

[38] G. Allaire, F. Jouve, and A.-M. Toader, "Structural optimization using sensitivity analysis and a level-set method," Journal of computational physics, vol. 194, no. 1, pp. 363-393, 2004.

[39] S. Osher and R. Fedkiw, Level set methods and dynamic implicit surfaces. Springer Science \& Business Media, 2006, vol. 153.

[40] F. Carpi, I. Anderson, S. Bauer, G. Frediani, G. Gallone, M. Gei, C. Graaf, C. Jean-Mistral, W. Kaal, G. Kofod et al., "Standards for dielectric elastomer transducers," Smart Materials and Structures, vol. 24 no. 10, p. 105025, 2015.

[41] R. Rivlin, "Large elastic deformations of isotropic materials iv. further developments of the general theory," Philosophical Transactions of the Royal Society of London. Series A, Mathematical and Physical Sciences, vol. 241, no. 835, pp. 379-397, 1948.

[42] X. Zhao and Z. Suo, "Method to analyze electromechanical stability of dielectric elastomers," Applied Physics Letters, vol. 91, no. 6, p. 061921, 2007.

[43] J. Huang, S. Shian, R. M. Diebold, Z. Suo, and D. R. Clarke, "The thickness and stretch dependence of the electrical breakdown strength of an acrylic dielectric elastomer," Applied Physics Letters, vol. 101, no. 12, p. 122905, 2012.

[44] S. Xu, Z. Yan, K.-I. Jang, W. Huang, H. Fu, J. Kim, Z. Wei, M. Flavin, J. McCracken, R. Wang et al., "Assembly of micro/nanomaterials into complex, three-dimensional architectures by compressive buckling," Science, vol. 347, no. 6218, pp. 154-159, 2015.

[45] M. A. McEvoy and N. Correll, "Materials that couple sensing, actuation, computation, and communication," Science, vol. 347, no. 6228, p. 1261689, 2015.

[46] E. Siéfert, E. Reyssat, J. Bico, and B. Roman, "Bio-inspired pneumatic shape-morphing elastomers," Nature materials, vol. 18 , no. 1 , p. 24 2019.

[47] X. Wang, X. Guo, J. Ye, N. Zheng, P. Kohli, D. Choi, Y. Zhang, Z. Xie, Q. Zhang, H. Luan et al., "Freestanding 3d mesostructures, functional devices, and shape-programmable systems based on mechanically induced assembly with shape memory polymers," Advanced Materials, vol. 31, no. 2, p. 1805615, 2019.
[48] A. Rafsanjani, Y. Zhang, B. Liu, S. M. Rubinstein, and K. Bertoldi, "Kirigami skins make a simple soft actuator crawl," Science Robotics, vol. 3, no. 15, p. eaar7555, 2018

[49] B. An, S. Miyashita, A. Ong, M. T. Tolley, M. L. Demaine, E. D. Demaine, R. J. Wood, and D. Rus, "An end-to-end approach to selffolding origami structures," IEEE Transactions on Robotics, vol. 34, no. 6, pp. 1409-1424, 2018

[50] Z. Zhakypov and J. Paik, "Design methodology for constructing multimaterial origami robots and machines," IEEE Transactions on Robotics, vol. 34, no. 1, pp. 151-165, 2018.

[51] O. Araromi, I. Gavrilovich, J. Shintake, S. Rosset, M. Richard, V. Gass, H. R. Shea et al., "Rollable multisegment dielectric elastomer minimum energy structures for a deployable microsatellite gripper," Mechatronics, IEEE/ASME Transactions on, vol. 20, no. 1, pp. 438-446, 2015.

[52] W.-B. Li, W.-M. Zhang, H.-X. Zou, Z.-K. Peng, and G. Meng, "A fast rolling soft robot driven by dielectric elastomer" IEEE/ASME Transactions on Mechatronics, vol. 23, no. 4, pp. 1630-1640, 2018.

[53] F. Carpi, G. Frediani, and D. De Rossi, "Hydrostatically coupled dielectric elastomer actuators," IEEE/ASME Transactions On Mechatronics, vol. 15 , no. 2, pp. 308-315, 2010.

[54] N. Wang, H. Guo, B. Chen, C. Cui, and X. Zhang, "Design of a rotary dielectric elastomer actuator using a topology optimization method based on pairs of curves," Smart Materials and Structures, vol. 27, no. 5, p. 055011, 2018

[55] E. Bortot, O. Amir, and G. Shmuel, "Topology optimization of dielectric elastomers for wide tunable band gaps," International Journal of Solids and Structures, vol. 143, pp. 262-273, 2018.

[56] F. Chen, W. Xu, H. Zhang, Y. Wang, J. Cao, M. Y. Wang, H. Ren, J. Zhu, and Y. Zhang, "Topology optimized design, fabrication, and characterization of a soft cable-driven gripper," IEEE Robotics and Automation Letters, vol. 3, no. 3, pp. 2463-2470, 2018.

[57] Y. Luo, M. Y. Wang, and Z. Kang, "Topology optimization of geometrically nonlinear structures based on an additive hyperelasticity technique," Computer methods in applied mechanics and engineering, vol. 286, pp. 422-441, 2015.

[58] H. Zhang, A. S. Kumar, F. Chen, J. Y. Fuh, and M. Y. Wang, "Topology optimized multimaterial soft fingers for applications on grippers, rehabilitation, and artificial hands," IEEE/ASME Transactions on Mechatronics, vol. 24, no. 1, pp. 120-131, 2019.

[59] J. Hiller and H. Lipson, "Automatic design and manufacture of soft robots," IEEE Transactions on Robotics, vol. 28, no. 2, pp. 457-466, 2012.

[60] G. Fang, C.-D. Matte, T.-H. Kwok, and C. C. Wang, "Geometrybased direct simulation for multi-material soft robots," in 2018 IEEE International Conference on Robotics and Automation (ICRA). IEEE, 2018, pp. 1-6.

[61] K. K. Choi and N.-H. Kim, Structural sensitivity analysis and optimization 1: linear systems. Springer Science \& Business Media, 2006.

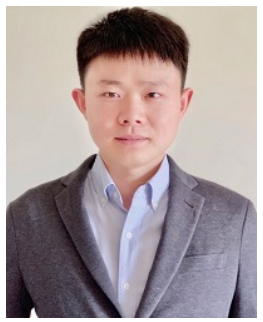

Feifei Chen (S'17-M'18) received the B.E. degree in mechanical engineering from University of Science and Technology of China in 2013, and Ph.D. degree in mechanical engineering from National University of Singapore in 2018. He joined Shanghai Jiao Tong University in 2018 and currently is an Assistant Professor (tenure-track) with the School of Mechanical Engineering.

His research interests include soft robotics, dielectric elastomer actuators, and topology optimization. 


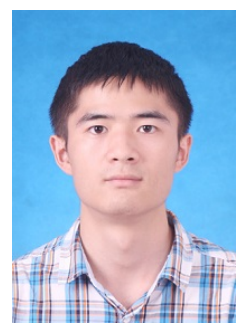

Kun Liu received the B.E. degree in materials processing and control from Huazhong University of Science and Technology, Wuhan, China, in 2017. $\mathrm{He}$ is currently working toward the M.S. degree in mechanical engineering at Shanghai Jiao Tong University, Shanghai, China.

His research interests include design, modeling, and control of soft robotics.

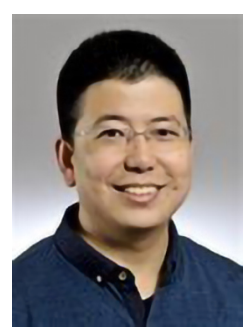

Yiqiang Wang received the B.E. degree and the Ph.D. degree in mechanics of engineering from Dalian University of Technology, in 2008 and 2014, respectively. After that, Dr. Wang worked as a Postdoc at the National University of Singapore and the Hong Kong University of Science and Technology. Since November 2017, Dr. Wang has worked at Technical University of Denmark as a H.C. Ørsted Postdoc (co-founded by Marie Skłodowska Curie Actions).

His research interests include innovative metamaterials design, structural topology optimization and interdisciplinary areas between computational mechanics and soft robots.

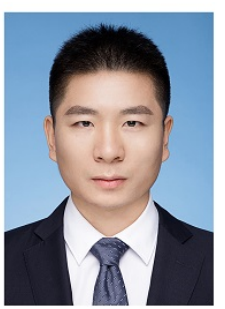

Jiang Zou received the B.E. degree (with honors) in mechanical engineering from the University of Science and Technology Beijing, Beijing, China, in 2014. He is currently working toward the Ph.D. degree in mechatronic engineering at Shanghai Jiao Tong University.

His research interests include design, modeling, and control of soft robotics.

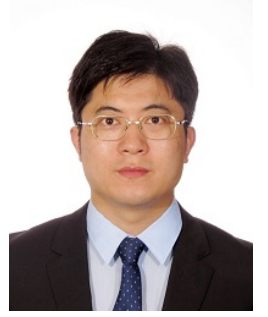

Guoying Gu (S'10-M'13) received the B.E. degree (with honors) in electronic science and technology, and the Ph.D. degree (with honors) in mechatronic engineering from Shanghai Jiao Tong University, Shanghai, China, in 2006 and 2012, respectively.

Since October 2012, Dr. Gu has worked at Shanghai Jiao Tong University, where he is currently appointed as a Professor of School of Mechanical Engineering. He was a Humboldt Postdoc Fellow with University of Oldenburg, Germany. He was a Visiting Scholar at Massachusetts Institute of Technology, National University of Singapore and Concordia University. His research interests include soft robotics, smart materials actuated systems, bioinspired robot design and motion control. He is the author or co-author of over 80 publications, which have appeared in Science Robotics, IEEE/ASME Trans., Advanced Functional Materials, Soft Robotics, etc., as book chapters and in conference proceedings.

Dr. $\mathrm{Gu}$ is the winner of multiple awards including Young Changjiang Scholar of the Ministry of Education, National Science Fund for Excellent Young Scholars, The first prize of natural science of Ministry of Education, Best Paper Award at the 2016 International Conference on Intelligent Robotics and Applications (ICIRA 2016) and 2011 IEEE International Conference on Information and Automation (IEEE-ICIA 2011). He is a member of the IEEE and ASME. Now he serves as Editorial Board Member of International Journal of Advanced Robotic Systems, and Topic Editor of Frontiers in Robotics and AI. He has also served as General Chair of 2017 International Symposium on Theory and Technology of Soft Robotics, and several international conferences/symposiums as Chair, Associate Editor or Program Committee Member.

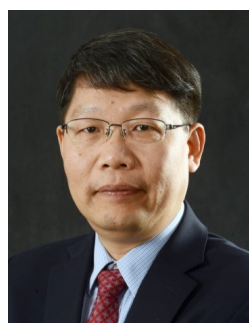

Xiangyang Zhu received the B.S. degree from the Department of Automatic Control Engineering, Nanjing Institute of Technology, Nanjing, China, in 1985, the M.Phil. degree in instrumentation engineering and the Ph.D. degree in control engineering, both from Southeast University, Nanjing, China, in 1989 and 1992, respectively. From 1993 to 1994 , he was a postdoctoral research fellow with Huazhong University of Science and Technology, Wuhan, China. He joined the Department of Mechanical Engineering, Southeast University, as an associate professor in 1995. Since June 2002, he has been with the School of Mechanical Engineering, Shanghai Jiao Tong University, Shanghai, China, where he is currently a chair professor and the director of the Robotics Institute. His research interests include robotic manipulation planning, neurointerfacing and neuro-prosthetics, and soft robotics. He has published more than 200 papers in international journals and conference proceedings.

Dr. Zhu has received a number of awards including the National Science Fund for Distinguished Young Scholars from NSFC in 2005, and the Cheung Kong Distinguished Professorship from the Ministry of Education in 2007. $\mathrm{He}$ is serving on the editorial board of IEEE Transactions on Cybernetics and Journal of Bionic Engineering. 